\title{
Fermionic condensate and the Casimir effect in cosmic string spacetime
}

\author{
A. Kh. Grigoryan ${ }^{1 *}$ A. R. Mkrtchyan ${ }^{1,2 \dagger}$ A. A. Saharian ${ }^{1,3 \ddagger}$ \\ ${ }^{1}$ Institute of Applied Problems in Physics NAS RA, \\ 25 Nersessian Street, 0014 Yerevan, Armenia \\ ${ }^{2}$ Tomsk Polytechnic University, 30 Lenin Avenue, 634050 Tomsk, Russia \\ ${ }^{3}$ Department of Physics, Yerevan State University, \\ 1 Alex Manoogian Street, 0025 Yerevan, Armenia
}

\begin{abstract}
We investigate combined effects of nontrivial topology, induced by a cosmic string, and boundaries on the fermionic condensate and the vacuum expectation value (VEV) of the energy-momentum tensor for a massive fermionic field. As geometry of boundaries we consider two plates perpendicular to the string axis on which the field is constrained by the MIT bag boundary condition. By using the Abel-Plana type summation formula, the VEVs in the region between the plates are decomposed into the boundary-free and boundaryinduced contributions for general case of the planar angle deficit. The boundary-induced parts in both the fermionic condensate and the energy-momentum tensor vanish on the cosmic string. Fermionic condensate is positive near the string and negative al large distances, whereas the vacuum energy density is negative everywhere. The radial stress is equal to the energy density. For a massless field, the boundary-induced contribution in the VEV of the energy-momentum tensor is different from zero in the region between the plates only and it does not depend on the coordinate along the string axis. In the region between the plates and at large distances from the string, the decay of the topological part is exponential for both massive and massless fields. This behavior is in contrast to that for the VEV of the energy-momentum tensor in the boundary-free geometry with the power law decay for a massless field. The vacuum pressure on the plates is inhomogeneous and vanishes at the location of the string. The corresponding Casimir forces are attractive.
\end{abstract}

PACS numbers: 03.70.+k, 98.80.Cq, 11.27.+d

\section{Introduction}

In a large number of field theoretical problems one needs to consider quantum fields in backgrounds with boundaries. The boundary conditions imposed on the field operator modify the spectrum of the zero-point fluctuations affecting the vacuum expectation values (VEVs) of physical observables. Among the most interesting manifestations of this influence is the Casimir effect

\footnotetext{
${ }^{*}$ E-mail: ashot.gr@gmail.com

${ }^{\dagger}$ E-mail: malpic@sci.am

${ }^{\ddagger}$ E-mail: saharian@ysu.am
} 
(for reviews see [1]). In 1948, Casimir showed that two neutral parallel conducting plates placed in the vacuum attract each other by the force inversely proportional to the fourth power of the distance between them. The appearance of the force was explained as a consequence of the change in the spectrum for the vacuum fluctuations of the electromagnetic field caused by the presence of the plates. Since the Casimir's original paper, the vacuum forces acting on the boundaries confining quantum fields have been investigated both theoretically and experimentally for other boundary geometries and also for scalar and fermionic fields. Similar effects arise in models with nontrivial spatial topology. In these models the boundary conditions imposed on the fields have topological origin.

Among the most interesting directions in the theory of the Casimir effect is its dependence on the geometry of the background spacetime. In particular, motivated by the possibility for the Casimir forces to serve as a stabilization mechanism for the radion field in braneworld models, the investigation of the Casimir effect in anti-de Sitter spacetime has attracted a great deal of attention (see, e.g., references given in [2]). In the present paper we consider a problem with two types of the sources for the fermionic vacuum polarization. The first one is topological and is induced by the presence of straight cosmic string and the second one is induced by two parallel conical boundaries. The cosmic strings are among the most interesting topological defects resulting from the symmetry braking phase transitions in grand unified theories [3]. In particular, they may have been created in the early Universe and provide an important link between particle physics and cosmology. A variant of the formation mechanism for cosmic strings has been proposed in models of brane inflation [4]. Although the recent observational data on the cosmic microwave background radiation (CMB) have ruled out cosmic strings as the primary source for primordial density perturbations, they are still candidates for the generation of a number of interesting physical effects. The latter include the gravitational lensing, the creation of small non-Gaussianities in the CMB, the generation of gravitational waves, highenergy cosmic rays, and gamma ray bursts. The conical space appears also as an effective background geometry in the long-wavelength description of condensed matter systems such as crystals, liquid crystals and quantum liquids [5]. Among condensed matter realizations of the fermionic model in the conical bulk are graphene nanocones. The latter are obtained from planar graphene sheet if one or more sectors with the angle $\pi / 3$ are excised and the remainder is joined [6]. The long-wavelength excitations of the electronic subsystem in graphene are described by Dirac-like theory with the Fermi velocity playing the role of the speed of light. In the case of graphene nanocones the background geometry for this theory is conical.

In the present paper we are interested in the influence of the nontrivial topology due to the cosmic string on the fermionic Casimir densities and the Casimir force in the geometry of two parallel plates with the MIT bag boundary condition. In order to have an exactly solvable problem, we will consider the idealized model for the straight cosmic string with flat spacetime everywhere except on the string where the curvature tensor has delta-type singularity. In this simplified model the exterior spacetime has a conical structure with the planar angle deficit related to the linear mass density. Among the interesting physical effects of the corresponding nontrivial topology, widely discussed in the literature, is the polarization of the vacuum for quantum fields (see, for instance, references given in [7]). Moreover, combined effects of the topology and boundaries in the geometry of the cosmic string have been considered as well. The analysis of the boundary-induced quantum effects in the cosmic string spacetime have been developed for scalar [8, 9, fermionic [10, 11, and electromagnetic fields [9, 12, 13, in the geometry of a coaxial cylindrical boundary. The Casimir force for a massless scalar fields subject to Dirichlet and Neumann boundary conditions in the setting of the conical piston has been investigated in [14]. The Casimir densities for scalar and electromagnetic fields induced by flat boundaries perpendicular to the string were considered in [15, 16, 17]. 
As local characteristics of the fermionic vacuum we will consider the fermionic condensate and the VEV of the energy-momentum tensor. The notion of the vacuum has a global nature and, as a consequence of this, these VEVs carry an important information about the global properties of the background spacetime. The fermionic condensate plays a crucial role in the models of chiral symmetry breaking and in considerations of the stability of the fermionic vacuum [18, 19]. In addition to describing the local properties of the vacuum, the VEV of the energy-momentum tensor acts as the source in semiclassical Einstein equations and is required in modelling the self-consistent dynamics involving the gravitational field.

The paper is organized as follows. In the next section we specify the bulk and boundary geometries and the boundary conditions imposed on the fermion field. The complete set of the positive- and negative-energy wavefunctions is presented. By making use of these modes, in section 3 we evaluate the fermionic condensate in the region between the plates. Various asymptotic limits of the general expression are discussed. Similar considerations for the VEV of the energy-momentum tensor are presented in section 4. The Casimir forces acting on the plates are investigated in section 5. The main results are summarized in section 6. Some details in the transformations of the components for the vacuum energy-momentum tensor are presented in Appendix A.

\section{Geometry of the problem and the mode functions}

We start with the description of the bulk and boundary geometries under consideration. The background geometry is generated by an infinitely long straight cosmic string along the $z$ axis. In the cylindrical coordinates $x^{\mu}=(t, r, \phi, z)$ the corresponding metric tensor reads $g_{\mu \nu}=$ $\operatorname{diag}\left(1,-1,-r^{2},-1\right)$. The difference from the Minkowskian spacetime comes from the planar angle deficit $2 \pi-\phi_{0}$ for the azimuthal angle $\phi, 0 \leqslant \phi \leqslant \phi_{0}$. In the discussion below we will also use the parameter $q=2 \pi / \phi_{0}$. In the weak-field approximation, one has the relation $2 \pi-\phi_{0}=8 \pi G \mu_{0}$, where $\mu_{0}$ is the linear mass density on the string and $G$ is the Newton gravitational constant.

For points outside the string core, the local geometry generated by the cosmic string is flat and coincides with that for the Minkowski spacetime. However, these two spacetimes have different global properties. The nontrivial topology coming from the cosmic string induces shifts in the VEVs of physical observables for quantum fields. Here we are interested in the influence of topology on the Casimir effect for a quantum fermionic field $\psi(x)$ in the geometry of two parallel plates perpendicular to the string axis. The field operator obeys the Dirac equation

$$
\left(i \gamma^{\mu} \nabla_{\mu}-m\right) \psi(x)=0
$$

with the covariant derivative $\nabla_{\mu}=\partial_{\mu}+\Gamma_{\mu}$ and with $\Gamma_{\mu}$ being the spin connection. In cylindrical coordinates with a planar angle deficit, for the Dirac $4 \times 4$ matrices $\gamma^{\mu}$ we will take the representation

$$
\gamma^{0}=\left(\begin{array}{cc}
1 & 0 \\
0 & -1
\end{array}\right), \gamma^{l}=\left(\begin{array}{cc}
0 & \beta^{l} \\
-\beta^{l} & 0
\end{array}\right)
$$

where $\beta^{l}, l=1,2,3$, are the Pauli matrices in the same coordinates. The latter are presented as

$$
\beta^{l}=(i / r)^{l-1}\left(\begin{array}{cc}
0 & (-1)^{l-1} e^{-i q \phi} \\
e^{i q \phi} & 0
\end{array}\right), \beta^{3}=\left(\begin{array}{cc}
1 & 0 \\
0 & -1
\end{array}\right),
$$

with $l=1,2$. 
We assume the presence of two boundaries located at $z=0$ and $z=a$ on which the field operator is constrained by the MIT bag boundary conditions:

$$
\left(1+i n_{\mu} \gamma^{\mu}\right) \psi(x)=0, \quad z=0, a,
$$

where $n_{\mu}$ is the outward-pointing normal to the boundary with respect to the region under consideration. In the discussion below we will consider the region $0 \leqslant z \leqslant a$ with $n_{\mu}=-\delta_{\mu 1}$ and $n_{\mu}=\delta_{\mu 1}$ for the boundaries at $z=0$ and $z=a$ respectively. In the regions $z \leqslant 0$ and $z \geqslant a$, the VEVs are obtained by the limiting transitions and they are the same as in the corresponding problem with a single boundary, discussed in [17. Note that the boundaries $z=0, a$ are twodimensional cones with the angle deficit $2 \pi-\phi_{0}$. The models with boundary conditions induced by the compactification of the cosmic string along its axis have been considered in [20].

We are interested in the investigation of the combined effects of topology and boundaries on the fermion condensate and on the VEV of the energy-momentum tensor. These quantities are expressed in the form of the sums over complete set of fermionic modes obeying the boundary conditions (2.4). So, our first step will be the determination of the complete set of the fermionic wavefunctions in the region between the boundaries. In [17] it has been shown that, in the geometry of a single plate at $z=0$, the positive-energy mode functions obeying the boundary condition (2.4) on that plate are presented as

$$
\psi_{\sigma}^{(+)}=C_{\sigma}^{(+)} e^{i(q j \phi-\omega t)}\left(\begin{array}{c}
f_{+}(z) J_{\beta}(\lambda r) e^{-i q \phi / 2} \\
i \frac{\epsilon_{j} s}{k} g_{+}(z) J_{\beta+\epsilon_{j}}(\lambda r) e^{i q \phi / 2} \\
\frac{1}{k} g_{-}(z) J_{\beta}(\lambda r) e^{-i q \phi / 2} \\
-i \epsilon_{j} s f_{-}(z) J_{\beta+\epsilon_{j}}(\lambda r) e^{i q \phi / 2}
\end{array}\right),
$$

where $J_{\beta}(x)$ is the Bessel function, $0 \leqslant \lambda<0, \epsilon_{j}=1$ for $j \geqslant 0$ and $\epsilon_{j}=-1$ for $j<0$ with $j= \pm 1 / 2, \pm 3 / 2, \ldots$, and

$$
\beta=q|j|-\epsilon_{j} / 2, \omega=\sqrt{\lambda^{2}+k^{2}+m^{2}} .
$$

In (2.5) we have defined the functions

$$
\begin{aligned}
& f_{ \pm}(z)=e^{i k z} \pm i \kappa_{s} e^{-i k z} \\
& g_{ \pm}(z)=(\omega \pm m) f_{ \pm}(z)+s \lambda f_{\mp}(z),
\end{aligned}
$$

with $k>0$ and

$$
\kappa_{s}=\frac{\omega+s \lambda}{k-i m}, s=-1,1
$$

In (2.5), $\sigma$ stands for the set of quantum numbers specifying the mode functions (see below).

Now we should impose on the modes (2.5) the boundary condition (2.4) at $z=a$. From the latter it follows that the eigenvalues of the quantum number $k$ are determined by the transcendental equation

$$
e^{2 i k a}=\frac{m-i k}{m+i k} .
$$

The latter can also be written in the form

$$
m a \sin (k a)+k a \cos (k a)=0 .
$$

This equation has an infinite number of positive solutions with respect to $k a$. We will denote them by $x_{n}=k a, n=1,2, \ldots, x_{n+1}>x_{n}$. For the eigenvalues of $k$ one has $k=k_{n}=x_{n} / a$. 
For the negative-energy mode functions, in a similar way, one has the expression

$$
\psi_{\sigma}^{(-)}=C_{\sigma}^{(-)} e^{i(q j \phi+\omega t)}\left(\begin{array}{c}
-\frac{1}{k} g_{-}(z) J_{\beta}(\lambda r) e^{-i q \phi / 2} \\
i \epsilon_{j} s f_{-}(z) J_{\beta+\epsilon_{j}}(\lambda r) e^{i q \phi / 2} \\
f_{+}(z) J_{\beta}(\lambda r) e^{-i q \phi / 2} \\
i \frac{\epsilon_{j} s}{k} g_{+}(z) J_{\beta+\epsilon_{j}}(\lambda r) e^{i q \phi / 2}
\end{array}\right)
$$

with the same notations as in (2.5) and with the same equation (2.10) for the eigenvalues of $k a$. It can be explicitly checked that the positive- and negative-energy modes (2.5) and (2.11) are orthogonal. The four-spinors $\psi_{\sigma}^{( \pm)}$are eigenfunctions of the projection of the total angular momentum along the cosmic string:

$$
\hat{J}_{3} \psi_{\sigma}^{( \pm)}=\left(-i \partial_{\phi}+i \frac{q}{2} r \gamma^{1} \gamma^{2}\right) \psi_{\sigma}^{( \pm)}=q j \psi_{\sigma}^{( \pm)} .
$$

Now, the set of quantum numbers for the modes are specified by $\sigma=(\lambda, j, n, s)$.

The coefficients $C_{\sigma}^{( \pm)}$in (2.5) and (2.11) are found from the orthonormality conditions

$$
\int d^{3} x \sqrt{|g|} \psi_{\sigma}^{( \pm) \dagger} \psi_{\sigma^{\prime}}^{( \pm)}=\delta\left(\lambda-\lambda^{\prime}\right) \delta_{n n^{\prime}} \delta_{j^{\prime} j} \delta_{s s^{\prime}},
$$

where the dagger denotes Hermitian conjugation and the integration goes over the region between the plates. By taking into account that for the Bessel function one has

$$
\int_{0}^{\infty} d r r J_{\beta}(\lambda r) J_{\beta}\left(\lambda^{\prime} r\right)=\frac{1}{\lambda} \delta\left(\lambda-\lambda^{\prime}\right)
$$

we get

$$
\left|C_{\sigma}^{( \pm)}\right|^{-2}=\frac{\phi_{0}}{\lambda} \frac{8 \omega(\omega+s \lambda) a}{k^{2}}\left[1-\frac{\sin (2 k a)}{2 k a}\right]
$$

with $k=x_{n} / a$.

\section{Fermionic condensate}

We start our investigation of the VEVs by the fermion condensate, defined as the expectation value $\langle 0|\bar{\psi} \psi| 0\rangle \equiv\langle\bar{\psi} \psi\rangle$, with $|0\rangle$ being the vacuum state and $\bar{\psi}=\psi^{\dagger} \gamma^{0}$ is the Dirac adjoint. Having the complete set of mode functions, the fermion condensate is evaluated by using the mode sum

$$
\langle\bar{\psi} \psi\rangle=\sum_{s= \pm 1} \sum_{j= \pm 1 / 2, \ldots} \sum_{n=1}^{\infty} \int_{0}^{\infty} d \lambda \bar{\psi}_{\sigma}^{(-)} \psi_{\sigma}^{(-)} .
$$

We assume that some regularization scheme is used to make the expression in the right-hand side finite (for example, a cutoff function is introduced or the arguments of the operators in the product are shifted). The choice of the specific scheme is not essential in the further discussion and we will not display it explicitly.

By making use of the expression (2.11) for the mode functions and the relations

$$
\sum_{j= \pm 1 / 2, \ldots} J_{\beta}^{2}(\lambda r)=\sum_{j= \pm 1 / 2, \ldots} J_{\beta+\epsilon_{j}}^{2}(\lambda r)=\sum_{j}\left[J_{q j-1 / 2}^{2}(\lambda r)+J_{q j+1 / 2}^{2}(\lambda r)\right]
$$

the fermionic condensate is presented in the form

$$
\langle\bar{\psi} \psi\rangle=-\frac{q m}{2 \pi a} \sum_{j} \int_{0}^{\infty} d \lambda \lambda \sum_{n=1}^{\infty} \frac{J_{q j-1 / 2}^{2}(\lambda r)+J_{q j+1 / 2}^{2}(\lambda r)}{\omega\left[1-\sin \left(2 x_{n}\right) /\left(2 x_{n}\right)\right]} h\left(k_{n}, z\right) .
$$


Here, $\omega=\sqrt{k_{n}^{2}+\lambda^{2}+m^{2}}$

$$
h(k, z)=2-\sum_{\eta= \pm 1}(1+\eta i k / m) e^{2 \eta i k z}
$$

and $\sum_{j}$ stands for the summation over $j=1 / 2,3 / 2, \cdots$.

By using the integral representation

$$
\omega=(2 / \sqrt{\pi}) \int_{0}^{\infty} d s e^{-\omega^{2} s^{2}},
$$

the integral over $\lambda$ in (3.3) is evaluated with the help of the formula [21]

$$
\int_{0}^{\infty} d \lambda \lambda J_{q j \pm 1 / 2}^{2}(\lambda r) e^{-\lambda^{2} s^{2}}=\frac{I_{q j \pm 1 / 2}\left(r^{2} / 2 s^{2}\right)}{2 s^{2} e^{r^{2} / 2 s^{2}}},
$$

where $I_{\nu}(x)$ is the modified Bessel function. As a result, the fermionic condensate is presented in the form

$$
\begin{aligned}
\langle\bar{\psi} \psi\rangle= & -\frac{q m}{(2 \pi)^{3 / 2} a r} \sum_{n=1}^{\infty} \frac{h\left(x_{n} / a, z\right)}{1-\sin \left(2 x_{n}\right) /\left(2 x_{n}\right)} \\
& \times \int_{0}^{\infty} d y y^{-1 / 2} e^{-\left(x_{n}^{2} / a^{2}+m^{2}\right) r^{2} / 2 y-y} \mathcal{I}(q, y),
\end{aligned}
$$

with the notation

$$
\mathcal{I}(q, y)=\sum_{j}\left[I_{q j-1 / 2}(y)+I_{q j+1 / 2}(y)\right] .
$$

As the next step in the evaluation of the fermionic condensate, for the series (3.8) we use the representation [11]

$$
\begin{aligned}
\mathcal{I}(q, x)= & \frac{2}{q} \sum_{l=0}^{p}(-1)^{l} \cos (\pi l / q) e^{x \cos (2 \pi l / q)} \\
& +\frac{2}{\pi} \cos (q \pi / 2) \int_{0}^{\infty} d u \frac{\sinh (q u / 2) \sinh (u / 2)}{\cosh (q u)-\cos (q \pi)} e^{-x \cosh u},
\end{aligned}
$$

where $p$ is the integer part of $q / 2, p=[q / 2]$. In (3.9), the prime on the sign of the sum means that the term $l=0$ and the term $l=p$ for even values of $q$ should be taken with the coefficients $1 / 2$. In the absence of the cosmic string one has $q=1$ and we get $\mathcal{I}(1, y)=e^{y}$. From here it follows that the contribution of the term $l=0$ in (3.9) to $\langle\bar{\psi} \psi\rangle$ coincides with the fermionic condensate in the region between two plates in the Minkowski spacetime. Substituting (3.9) into (3.7), the integral over $y$ is explicitly evaluated and one finds the following representation

$$
\begin{aligned}
\langle\bar{\psi} \psi\rangle= & -\frac{m}{2 \pi a r} \sum_{n=1}^{\infty} \frac{h\left(x_{n} / a, z\right)}{1-\sin \left(2 x_{n}\right) /\left(2 x_{n}\right)}\left[\sum_{l=0}^{p}(-1)^{l} \frac{c_{l}}{s_{l}} e^{-2 r s_{l} \sqrt{k_{n}^{2}+m^{2}}}\right. \\
& \left.+\frac{2 q}{\pi} \int_{0}^{\infty} d x \frac{\cos (q \pi / 2) \sinh (q x) \sinh x}{\cosh (2 q x)-\cos (q \pi)} \frac{e^{-2 r \sqrt{k_{n}^{2}+m^{2}} \cosh x}}{\cosh x}\right],
\end{aligned}
$$

with the notations

$$
s_{l}=\sin (\pi l / q), c_{l}=\cos (\pi l / q) .
$$


For the general case of a massive field, the eigenvalues $k_{n}$ in (3.10) are given implicitly, as roots of (2.10), and this form for the fermionic condensate is not convenient for the further investigation of its properties. For the summation over these eigenvalues we use the formula

$$
\sum_{n=1}^{\infty} \frac{\pi f\left(x_{n}\right)}{1-\sin \left(2 x_{n}\right) /\left(2 x_{n}\right)}=-\frac{\pi m a f(0)}{2(m a+1)}+\int_{0}^{\infty} d x f(x)-i \int_{0}^{\infty} d x \frac{f(i x)-f(-i x)}{\frac{x+m a}{x-m a} e^{2 x}+1},
$$

that is a consequence of the generalized Abel-Plana summation formula [22]. For the series in (3.10) the corresponding function is given by the expression

$$
f(x)=h(x / a, z) e^{-2 b r \sqrt{x^{2} / a^{2}+m^{2}}} .
$$

For this function $f(0)=0$ and the first term in the right-hand side of (3.12) is absent. The first integral in (3.12) is evaluated by using the formula

$$
\int_{m}^{\infty} d x \sin \left(2 y \sqrt{x^{2}-m^{2}}\right) e^{-2 z x}=2 y m^{2} f_{1}\left(2 m \sqrt{z^{2}+y^{2}}\right)
$$

with the notation

$$
f_{\nu}(x)=K_{\nu}(x) / x^{\nu}
$$

and the relation

$$
\int_{0}^{\infty} d x \sum_{\eta= \pm 1}(m+\eta i x) e^{2 \eta i x z} e^{-2 y \sqrt{x^{2}+m^{2}}}=\left(2 m+\partial_{z}\right) \int_{m}^{\infty} d x \sin \left(2 y \sqrt{x^{2}-m^{2}}\right) e^{-2 x z} .
$$

In deriving (3.16) we have rotated the integration contour of the left-hand side in the complex $x$-plane by the angle $\pi / 2$ for $\eta=1$ and by $-\pi / 2$ for $\eta=-1$. As a result one finds the representation

$$
\frac{\pi}{a} \sum_{n=1}^{\infty} \frac{h\left(x_{n} / a, z\right) e^{-2 y \sqrt{x_{n}^{2} / a^{2}+m^{2}}}}{1-\sin \left(2 x_{n}\right) /\left(2 x_{n}\right)}=4 m^{2} y\left[f_{1}(2 m y)-F(2 m z, 2 m y)\right]-\frac{y}{m} B(z, y),
$$

where we have defined the functions

$$
F(x, y)=f_{1}\left(\sqrt{x^{2}+y^{2}}\right)-x f_{2}\left(\sqrt{x^{2}+y^{2}}\right),
$$

and

$$
B(z, y)=\frac{2}{y} \int_{m}^{\infty} d x \frac{\sin \left(2 y \sqrt{x^{2}-m^{2}}\right)}{\frac{x+m}{x-m} e^{2 a x}+1}\left[2 m-\sum_{\eta= \pm 1}(m+\eta x) e^{2 \eta z x}\right] .
$$

Here, the relation $f_{\nu}^{\prime}(x)=-x f_{\nu+1}(x)$ is used for the function (3.15).

Substituting (3.17) into (3.10), the fermionic condensate is decomposed into three contributions. The first one comes from the term with $f_{1}(2 m y)$ in (3.17). This contribution does not depend on $z$ and on $a$ and corresponds to the fermionic condensate in the boundary-free cosmic string spacetime. For points away from the boundaries, the only divergence in the fermionic condensate is contained in the $l=0$ term of this contribution. The latter coincides with the fermionic condensate in boundary-free Minkowski spacetime. The renormalization is reduced to omitting this term. As a result, the renormalized fermionic condensate in the boundary-free cosmic string geometry is presented as

$$
\begin{aligned}
\langle\bar{\psi} \psi\rangle_{\mathrm{s}}= & -\frac{2 m^{3}}{\pi^{2}}\left[\sum_{l=1}^{p}(-1)^{l} c_{l} f_{1}\left(2 m r s_{l}\right)+\frac{2 q}{\pi} \cos \left(\frac{q \pi}{2}\right)\right. \\
& \left.\times \int_{0}^{\infty} d x \frac{\sinh (q x) \sinh (x) f_{1}(2 m r \cosh x)}{\cosh (2 q x)-\cos (q \pi)}\right] .
\end{aligned}
$$


This expression has been previously derived in [17] (for the generalization in the geometry of cosmic string with a magnetic flux see [7]). For a massless field the boundary-free part (3.20) vanishes, $\langle\bar{\psi} \psi\rangle_{\mathrm{s}}=0$. For massive fields, the condensate $\langle\bar{\psi} \psi\rangle_{\mathrm{s}}$ is positive everywhere. It diverges on the string as $1 / r^{2}$ and is exponentially suppressed at large distances, $m r \gg 1$.

The contribution of the term with $F(2 m z, 2 m y)$ in (3.17) to the fermionic condensate does not depend on $a$ whereas the contribution of the last term in the right-hand side of (3.17) vanishes in the limit $a \rightarrow \infty$. From here it follows that the part

$$
\begin{aligned}
\langle\bar{\psi} \psi\rangle_{\mathrm{b}}^{(1)}= & \frac{2 m^{3}}{\pi^{2}}\left[\sum_{l=0}^{p}(-1)^{l} c_{l} F\left(2 m z, 2 m r s_{l}\right)+\frac{2 q}{\pi} \cos \left(\frac{q \pi}{2}\right)\right. \\
& \left.\times \int_{0}^{\infty} d x \frac{\cos (q \pi / 2) \sinh (q x) \sinh x}{\cosh (2 q x)-\cos (q \pi)} F(2 m z, 2 m r \cosh x)\right],
\end{aligned}
$$

is induced by the presence of the boundary at $z=0$ when the second boundary is absent. Hence, in the geometry of a single boundary at $z=0$ the renormalized fermionic condensate is decomposed as

$$
\langle\bar{\psi} \psi\rangle^{(1)}=\langle\bar{\psi} \psi\rangle_{\mathrm{s}}+\langle\bar{\psi} \psi\rangle_{\mathrm{b}}^{(1)} .
$$

This result, with (3.20) and (3.21), coincides with that obtained in [17. Hence, in the region between two boundaries the fermionic condensate is written in the form

$$
\begin{aligned}
\langle\bar{\psi} \psi\rangle= & \langle\bar{\psi} \psi\rangle^{(1)}+\frac{1}{2 \pi^{2}}\left[\sum_{l=0}^{p}(-1)^{l} c_{l} B\left(z, r s_{l}\right)\right. \\
& \left.+\frac{2 q}{\pi} \cos \left(\frac{q \pi}{2}\right) \int_{0}^{\infty} d x \frac{\sinh (q x) \sinh (x) B(z, r \cosh x)}{\cosh (2 q x)-\cos (q \pi)}\right],
\end{aligned}
$$

where the last term is induced by the presence of the second boundary at $z=a$.

By taking into account that

$$
F(2 m z, 2 m y)=-\frac{m^{-3}}{2 y} \int_{m}^{\infty} d x \frac{x-m}{e^{2 z x}} \sin \left(2 y \sqrt{x^{2}-m^{2}}\right)
$$

and combining the single plate-induced part $\langle\bar{\psi} \psi\rangle_{\mathrm{b}}^{(1)}$ with the last term in (3.23) , we find the decomposition

$$
\langle\bar{\psi} \psi\rangle=\langle\bar{\psi} \psi\rangle_{\mathrm{s}}+\langle\bar{\psi} \psi\rangle_{\mathrm{b}}
$$

Here, the boundary-induced contribution to the fermionic condensate in the region between the plates is given by

$$
\begin{aligned}
\langle\bar{\psi} \psi\rangle_{\mathrm{b}}= & \frac{1}{2 \pi^{2}}\left[\sum_{l=0}^{p}(-1)^{l} c_{l} C\left(z, r s_{l}\right)+\frac{2 q}{\pi} \cos \left(\frac{q \pi}{2}\right)\right. \\
& \left.\times \int_{0}^{\infty} d x \frac{\sinh (q x) \sinh (x) C(z, r \cosh x)}{\cosh (2 q x)-\cos (q \pi)}\right],
\end{aligned}
$$

with the function

$$
C(z, y)=\frac{2}{y} \int_{m}^{\infty} d x \frac{\sin \left(2 y \sqrt{x^{2}-m^{2}}\right)}{\frac{x+m}{x-m} e^{2 a x}+1}\left[2 m-(m+x)\left(e^{2 z x}+e^{2(a-z) x}\right)\right] .
$$

Taking the limit $a \rightarrow \infty, z \rightarrow \infty$, with fixed $a-z$, and using the relation (3.24), we can see that from the second term in the right-hand side of (3.23) the boundary-induced part is obtained 
for a single plate at $z=a$. The latter is given by the expression (3.21) with the replacement $z \rightarrow a-z$.

For a massless field, $m=0$, from (3.27) one gets

$$
C(z, y)=\frac{\pi}{a y} \partial_{z} \frac{\sinh (\pi y / a) \cos (\pi z / a)}{\cosh (2 \pi y / a)-\cos (2 \pi z / a)} .
$$

In this special case the boundary-free part vanishes and for the single plate contribution one has (see [17])

$$
\langle\bar{\psi} \psi\rangle^{(1)}=-\frac{q}{4 \pi^{2} r z^{2}} \frac{u^{q}}{u^{2 q}-1}\left(1+\frac{q z}{\sqrt{r^{2}+z^{2}}} \frac{u^{2 q}+1}{u^{2 q}-1}\right),
$$

with the notation $u=z / r+\sqrt{1+z^{2} / r^{2}}$.

For $q=1$, in (3.26) the $l=0$ term survives only and we get the corresponding result for two parallel plates in Minkowski bulk:

$$
\langle\bar{\psi} \psi\rangle_{\mathrm{M}}=\frac{1}{\pi^{2}} \int_{m}^{\infty} d x \frac{\sqrt{x^{2}-m^{2}}}{\frac{x+m}{x-m} e^{2 a x}+1}\left[2 m-(m+x)\left(e^{2 z x}+e^{2(a-z) x}\right)\right] .
$$

In this case the fermionic condensate is negative. The remaining part in (3.26) is induced by the presence of the cosmic string. In particular, for a massless field the Minkowskian part is simplified to

$$
\langle\bar{\psi} \psi\rangle_{\mathrm{M}}=-\pi \frac{2-\sin ^{2}(\pi z / a)}{8 a^{3} \sin ^{3}(\pi z / a)} .
$$

For points near the plate $z=0$, to the leading order, we get

$$
\langle\bar{\psi} \psi\rangle_{\mathrm{M}} \approx-\frac{1}{4 \pi^{2} z^{3}} .
$$

This expression gives the leading term in the asymptotic expansion for the fermionic condensate near the plate for massive fields as well. Moreover, to the leading order and under the condition $z \ll r$, the expression in the right-hand side of (3.32) gives the asymptotic in the presence of the cosmic string: $\langle\bar{\psi} \psi\rangle \approx-1 /\left(4 \pi^{2} z^{3}\right)$. The generalization of (3.30) for arbitrary number of spatial dimensions is given in [23]. The fermionic condensate for the geometry of two parallel on AdS bulk has been investigated in [24].

For points outside the plates, $z \neq 0, a$, the boundary-induced contribution (3.26) is finite on the string. By taking into account that $\lim _{y \rightarrow 0} C(z, y)=4 \pi^{2}\langle\bar{\psi} \psi\rangle_{M}$, we obtain

$$
\langle\bar{\psi} \psi\rangle_{\mathrm{b}, r=0}=2\langle\bar{\psi} \psi\rangle_{M}\left[\sum_{l=0}^{p}(-1)^{l} c_{l}+\frac{2 q}{\pi} \cos \left(\frac{q \pi}{2}\right) \int_{0}^{\infty} d x \frac{\sinh (q x) \sinh x}{\cosh (2 q x)-\cos (q \pi)}\right] .
$$

The expression in the square brackets vanishes and, hence, the boundary-induced part in the fermionic condensate vanishes on the string. From here it follows that for points near the string and for a massive field the total fermionic condensate is dominated by the boundary-free contribution and is positive.

Now let us consider the asymptotic behavior of the fermionic condensate at large distances from the string, $r \gg a, m^{-1}$. Here we need the asymptotic of the function $C(z, y)$ for large values of the second argument. It is obtained by taking into account that for large $y$ the dominant contribution to the integral in (3.27) comes from the region near the lower limit of the integration. To the leading order, the integral is expressed in terms of the Macdonald function. By using the corresponding asymptotic expression for large arguments, we can see that

$$
C(z, y) \approx \sqrt{\pi} m^{3}\left[e^{2 z m}+e^{2(a-z) m}-1\right] \frac{e^{-2 m y}}{(m y)^{3 / 2}}
$$


for $y \gg a, m^{-1}$. At large distances, the leading contribution in the boundary-induced part of the fermionic condensate comes from the term $l=0$ which coincides with $\langle\bar{\psi} \psi\rangle_{\mathrm{M}}$. For $q>2$, the next to the leading term comes from the term $l=1$ in (3.26). Adding the asymptotic for the boundary-free contribution one gets

$$
\langle\bar{\psi} \psi\rangle \approx\langle\bar{\psi} \psi\rangle_{\mathrm{M}}-\left[e^{2 z m}+e^{2(a-z) m}-2\right] \frac{m^{3} \cos (\pi / q) e^{-2 m r \sin (\pi / q)}}{2(\pi m r \sin (\pi / q))^{3 / 2}} .
$$

The topological part, given by the second term in the right-hand side, is negative. For a massless field the asymptotic expression is directly obtained by using (3.28):

$$
\langle\bar{\psi} \psi\rangle \approx\langle\bar{\psi} \psi\rangle_{\mathrm{M}}+\frac{\cot (\pi / q) \sin (\pi z / a)}{2 \pi a^{2} r e^{\pi r \sin (\pi / q) / a}} .
$$

Note that in this case the topological part is positive. For $q \leqslant 2$, the decay of the topological part in the boundary-induced fermion condensate at large distances from the string is stronger, like $e^{-2 m r}$ and $e^{-\pi r / a}$ for the massive and massless cases, respectively. Hence, at large distances from the string, to the leading order, the fermionic condensate coincides with that for the plates in Minkowski bulk and is negative. As we have shown before, the condensate is positive near the string. As a consequence, the fermionic condensate vanishes for some intermediate value of the radial coordinate $r$. Note that at large distances from the string the topological part in the region between the plates is suppressed exponentially for both massive and massless fields.

Let us denote the part in the fermionic condensate induced by the cosmic string as $\langle\bar{\psi} \psi\rangle_{\mathrm{t}}$. It is given by $\langle\bar{\psi} \psi\rangle_{\mathrm{t}}=\langle\bar{\psi} \psi\rangle-\langle\bar{\psi} \psi\rangle_{\mathrm{M}}$ and can be termed as topological part. The corresponding expression is obtained from (3.25) omitting the $l=0$ term in the boundary-induced contribution (3.26). For a massless field, by taking into account that for $y \neq 0$ one has $C(0, y)=C(a, y)=0$ (see (3.28) ), we conclude that the topological part in the fermionic condensate vanishes on the plates. The same is the case for a massive field. In order to show that, we firstly consider the topological part in the boundary-induced contribution (3.26). This part is obtained from (3.26) omitting the term $l=0$. For the evaluation of the topological part we cannot directly put $z=0, a$ in the corresponding expression. The limit $z \rightarrow 0, a$ should be taken after the evaluation

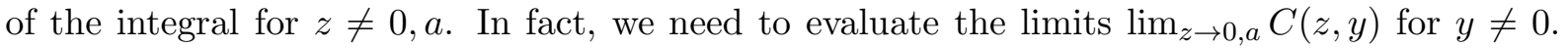
These limits are the same and we will consider the case $z \rightarrow 0$ only. The evaluation procedure is simplified if we introduce in the integrand the factor $e^{-\alpha \sqrt{x^{2}-m^{2}}}$ with $\alpha>0$ and take the limit $\alpha \rightarrow 0$ after the evaluation of the integral. With this factor we can directly put $z=0$ in the integrand. In this way it can be seen that

$$
\lim _{z \rightarrow 0} C(z, y)=4 m^{3} f_{1}(2 m y)
$$

for $y \neq 0$. Comparing with the boundary-free fermionic condensate (3.20), we see that the boundary-induced contribution in the topological part exactly cancels the boundary-free part and the topological part of the fermionic condensate vanishes on the plates. This feature is seen from figure 1, where, for a massless field and for the value of the parameter $q=3$, we have plotted the topological part in the fermionic condensate, $a^{3}\langle\bar{\psi} \psi\rangle_{\mathrm{t}}$, as a function of the distance from the string and of the distance from the plate at $z=0$.

An alternative representation for the fermionic condensate is obtained by the application of the summation formula (3.12) to the series over $n$ in (3.3). The part with the first integral in the right-hand side of (3.12) will give the fermion condensate for the geometry of a single plate at $z=0$. In the corresponding expression we first separate the boundary-free contribution, obtained from the first term in the right-hand side of (3.4), and in the remaining part we rotate the integration contour over $k$ by the angle $\pi / 2(-\pi / 2)$ for the term with $e^{2 i k z}\left(e^{-2 i k z}\right)$. 


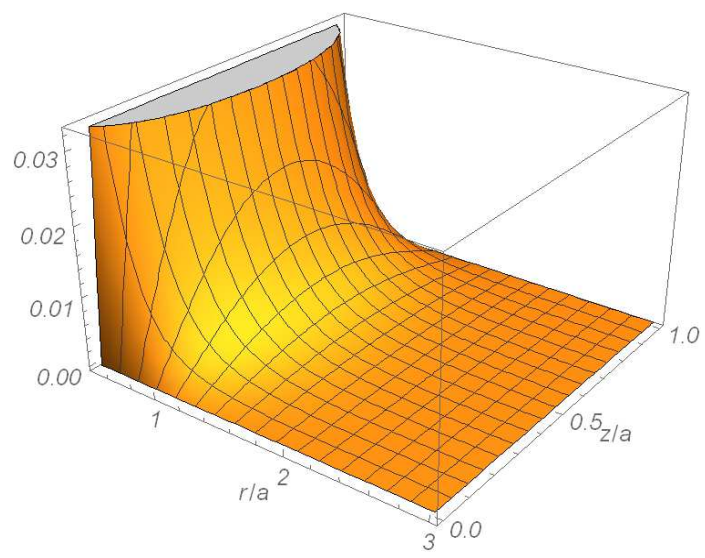

Figure 1: The topological part in the fermionic condensate, $a^{3}\langle\bar{\psi} \psi\rangle_{\mathrm{cs}}$, for a massless field as a function of the distances from the string and from the left plate. For the planar angle deficit we have taken the value corresponding to $q=3$.

Combining with the term coming from the second integral in (3.12), for the fermionic condensate in the region between the plates we obtain the representation

$$
\begin{aligned}
\langle\bar{\psi} \psi\rangle= & \langle\bar{\psi} \psi\rangle_{\mathrm{s}}-\frac{q}{\pi^{2}} \sum_{j} \int_{0}^{\infty} d \lambda \lambda\left[J_{q j-1 / 2}^{2}(\lambda r)+J_{q j+1 / 2}^{2}(\lambda r)\right] \\
& \times \int_{\sqrt{\lambda^{2}+m^{2}}}^{\infty} d x \frac{(x+m)\left[e^{2 x z}+e^{2(a-z) x}\right]-2 m}{\sqrt{x^{2}-\lambda^{2}-m^{2}}\left(\frac{x+m}{x-m} e^{2 a x}+1\right)} .
\end{aligned}
$$

As is seen from this formula, the boundary-induced contribution to the fermionic condensate, given by the second term in the right-hand side of (3.38), is always negative. In the limit $r \rightarrow 0$, the dominant contribution to the boundary-induced part in (3.38) comes from the term $j=1 / 2$ and, by using the asymptotic for the Bessel function for small values of the argument, to the leading order we get

$$
\begin{aligned}
\langle\bar{\psi} \psi\rangle \approx & \langle\bar{\psi} \psi\rangle_{\mathrm{s}}-\frac{\pi^{-3 / 2}(r / 2)^{q-1}}{\Gamma(q / 2) \Gamma((q+1) / 2)} \int_{m}^{\infty} d x \frac{\left(x^{2}-m^{2}\right)^{q / 2}}{\frac{x+m}{x-m} e^{2 a x}+1} \\
& \times\left[(x+m)\left(e^{2 x z}+e^{2(a-z) x}\right)-2 m\right] .
\end{aligned}
$$

For $q=1$ this asymptotic formula is reduced to the exact result (3.30).

In a number of field theoretical models, the Lagrangian, in addition to the fermionic mass term $m \bar{\psi} \psi$, contains other terms involving the product $\bar{\psi} \psi$. An example is the fermionc field nonminimally coupled to gravity with the interaction term $\xi R \bar{\psi} \psi$ (see, e.g., [25]), where $R$ is the scalar curvature for the background spacetime and $\xi$ is a constant with the dimension of the inverse mass. The formation of nonzero fermionic condensate induces an additional term $\xi R\langle\bar{\psi} \psi\rangle$ in the Lagrangian for the gravitational field. This leads to the shift (in general, spacedependent) in the gravitational constant. Another example is the fermionic field interacting with a scalar field $\varphi$ through the interaction Lagrangian proportional to $\varphi \bar{\psi} \psi$. The interaction terms of this form appear also in semibosonized versions of the four-fermion interaction models of the Gross-Neveu and Nambu-Jona-Lasinio type. The effects of the background geometry, topology and boundaries in these models have been discussed in [19, 26]. 


\section{Energy-momentum tensor}

The mode sum for the VEV of the energy-momentum tensor, $\left\langle 0\left|T_{\mu \nu}\right| 0\right\rangle \equiv\left\langle T_{\mu \nu}\right\rangle$, is given by the expression

$$
\left\langle T_{\mu \nu}\right\rangle=\frac{i}{2} \sum_{s= \pm 1} \sum_{j= \pm 1 / 2, \ldots} \sum_{n=1}^{\infty} \int_{0}^{\infty} d \lambda\left[\bar{\psi}_{\sigma}^{(-)} \gamma_{(\mu} \nabla_{\nu)} \psi_{\sigma}^{(-)}-\left(\nabla_{(\mu} \bar{\psi}_{\sigma}^{(-)}\right) \gamma_{\nu)} \psi_{\sigma}^{(-)}\right]
$$

where $\gamma_{\mu}=g_{\mu \nu} \gamma^{\nu}$ and the brackets enclosing the indices mean the symmetrization. Similar to the case of the condensate, we assume that some regularization procedure is used for the righthand side of (4.1) without explicitly writing it. Inserting the expression for the negative-energy mode functions, we can see that the off-diagonal components vanish. The VEVs for the diagonal components are presented in the form (no summation over $\nu$ ):

$$
\left\langle T_{\nu}^{\nu}\right\rangle=\frac{q}{\pi a} \sum_{j} \sum_{n=1}^{\infty} \int_{0}^{\infty} d \lambda \frac{\lambda^{3}}{\omega} \frac{g_{q j-1 / 2}^{(\nu)}(\lambda r) h_{\nu}\left(k_{n}, z\right)}{1-\sin \left(2 x_{n}\right) /\left(2 x_{n}\right)}
$$

with the notations

$$
\begin{aligned}
& g_{\beta}^{(0)}(y)=-\frac{\omega^{2}}{k^{2}} g_{\beta}^{(3)}(y)=-\frac{\omega^{2}}{\lambda^{2}}\left[J_{\beta}^{2}(y)+J_{\beta+1}^{2}(y)\right], \\
& g_{\beta}^{(1)}(y)=J_{\beta}^{2}(y)+J_{\beta+1}^{2}(y)-\frac{2 q j}{y} J_{\beta}(y) J_{\beta+1}(y), \\
& g_{\beta}^{(2)}(y)=\frac{2 q j}{y} J_{\beta}(y) J_{\beta+1}(y),
\end{aligned}
$$

and

$$
h_{\nu}(k, z)=1-\frac{1-\delta_{3}^{\nu}}{2} \sum_{\eta= \pm 1} \frac{m e^{2 \eta i k z}}{m-\eta i k} .
$$

Note that the axial stress does not depend on the coordinate $z$. We can see that the VEVs (4.2) obey the trace relation

$$
\left\langle T_{\nu}^{\nu}\right\rangle=m\langle\bar{\psi} \psi\rangle
$$

In Appendix we show that the diagonal components of the vacuum energy-momentum tensor are transformed to the form

$$
\begin{aligned}
\left\langle T_{\nu}^{\nu}\right\rangle= & -\frac{1}{4 \pi a} \sum_{n=1}^{\infty} \frac{1}{1-\sin \left(2 x_{n}\right) /\left(2 x_{n}\right)}\left[\sum_{l=0}^{p}(-1)^{l} c_{l} D_{\nu}\left(z, r s_{l}, k_{n}\right)\right. \\
& \left.+\frac{2 q}{\pi} \cos \left(\frac{q \pi}{2}\right) \int_{0}^{\infty} d u \frac{\sinh (q u) \sinh (u) D_{\nu}\left(z, r \cosh u, k_{n}\right)}{\cosh (2 q u)-\cos (q \pi)}\right],
\end{aligned}
$$

with the notation

$$
D_{\nu}(z, y, k)=\hat{D}_{\nu} e^{-2 y \sqrt{k^{2}+m^{2}}} h_{\nu}(k, z),
$$

where we have defined the operators

$$
\begin{aligned}
& \hat{D}_{0}=\hat{D}_{1}=y^{-3}\left(y \partial_{y}-1\right) \\
& \hat{D}_{2}=2 y^{-3}\left(1-y \partial_{y}+\frac{1}{2} y^{2} \partial_{y}^{2}\right) \\
& \hat{D}_{3}=y^{-1}\left(4 m^{2}-\partial_{y}^{2}\right)
\end{aligned}
$$


For these operators we have the relation

$$
\sum_{\nu=0}^{3} \hat{D}_{\nu}=4 m^{2} / y
$$

As is seen from (4.7), the radial stress is equal to the energy density.

Now we apply to the series over $n$ in (4.6) the summation formula (3.12) with the function

$$
f(x)=e^{-2 y \sqrt{x^{2} / a^{2}+m^{2}}} h_{\nu}(x / a, z) .
$$

As a result, the VEVs are decomposed as

$$
\begin{aligned}
\left\langle T_{\nu}^{\nu}\right\rangle= & \left\langle T_{\nu}^{\nu}\right\rangle^{(1)}+\frac{1}{2 \pi^{2}}\left[\sum_{l=0}^{p}(-1)^{l} c_{l} B_{\nu}\left(z, r s_{l}\right)\right. \\
& \left.+\frac{2 q}{\pi} \cos \left(\frac{q \pi}{2}\right) \int_{0}^{\infty} d u \frac{\sinh (q u) \sinh (u) B_{\nu}(z, r \cosh u)}{\cosh (2 q u)-\cos (q \pi)}\right],
\end{aligned}
$$

with the function

$$
B_{\nu}(z, y)=\hat{D}_{\nu} \int_{m}^{\infty} d x \frac{\sin \left(2 y \sqrt{x^{2}-m^{2}}\right)}{\frac{x+m}{x-m} e^{2 a x}+1}\left(1-\frac{1-\delta_{3}^{\nu}}{2} \sum_{\eta= \pm 1} \frac{m e^{2 \eta x z}}{m-\eta x}\right) .
$$

The first term in the right-hand side of (4.10) comes from the first integral in (3.12) and is given by the expression

$$
\begin{aligned}
\left\langle T_{\nu}^{\nu}\right\rangle^{(1)}= & -\frac{1}{4 \pi^{2}}\left[\sum_{l=0}^{p}(-1)^{l} c_{l} B_{\nu}^{(0)}\left(z, r s_{l}\right)+\frac{2 q}{\pi} \cos \left(\frac{q \pi}{2}\right)\right. \\
& \left.\times \int_{0}^{\infty} d u \frac{\sinh (q u) \sinh (u) B_{\nu}^{(0)}(z, r \cosh u)}{\cosh (2 q u)-\cos (q \pi)}\right],
\end{aligned}
$$

where

$$
B_{\nu}^{(0)}(z, y)=\hat{D}_{\nu} \int_{0}^{\infty} d x e^{-2 y \sqrt{x^{2}+m^{2}}} h_{\nu}(x, z) .
$$

The contribution (4.12) does not depend on $a$, whereas the second term in the right-hand side of (4.10) vanishes in the limit $a \rightarrow \infty$. From here it follows that the part $\left\langle T_{\nu}^{\nu}\right\rangle^{(1)}$ corresponds to the VEV in the geometry of a single plate at $z=0$.

The further transformation of the contribution $\left\langle T_{\nu}^{\nu}\right\rangle^{(1)}$ is similar to that we have used for $\langle\bar{\psi} \psi\rangle^{(1)}$ in the previous section. We decompose $\left\langle T_{\nu}^{\nu}\right\rangle^{(1)}$ into two parts coming from the first and second terms in the right-hand side of (4.4). The part with $h_{\nu}(x, z) \rightarrow 1$ corresponds to the VEV in the boundary-free cosmic string spacetime. In this part the corresponding integral (4.13) is expressed in terms of the Macdonald function and the renormalization is reduced to omitting the $l=0$ term. The corresponding renormalizaed VEV is presented as (no summation over $\nu$ )

$$
\begin{aligned}
\left\langle T_{\nu}^{\nu}\right\rangle_{\mathrm{s}}= & \frac{2 m^{4}}{\pi^{2}}\left[\sum_{l=1}^{p}(-1)^{l} c_{l} F_{\nu}^{(0)}\left(2 m r s_{l}\right)+\frac{2 q}{\pi} \cos \left(\frac{q \pi}{2}\right)\right. \\
& \left.\times \int_{0}^{\infty} d x \frac{\sinh (q x) \sinh (x) F_{\nu}^{(0)}(2 m r \cosh x)}{\cosh (2 q x)-\cos (q \pi)}\right],
\end{aligned}
$$


where we have defined the functions

$$
\begin{aligned}
& F_{0}^{(0)}(y)=F_{1}^{(0)}(y)=F_{3}^{(0)}(y)=f_{2}(y), \\
& F_{2}^{(0)}(y)=-3 f_{2}(y)-f_{1}(y),
\end{aligned}
$$

with the notation (3.15). The corresponding energy density is negative everywhere. An alternative integral representation is given in [10]. The fermionic VEVs $\left\langle T_{\nu}^{\nu}\right\rangle_{\mathrm{s}}$ in the special case $q<2$ have been previously investigated in [27]. For a massless field the renormalized VEV of the energy-momentum tensor in the boundary-free cosmic string spacetime was found in [28] and is obtained from (4.14) taking the limit $m \rightarrow 0$ :

$$
\left\langle T_{\nu}^{\nu}\right\rangle_{\mathrm{s}}=-\frac{\left(q^{2}-1\right)\left(7 q^{2}+17\right)}{2880 \pi^{2} r^{4}} \operatorname{diag}(1,1,-3,1) .
$$

Note that in the boundary-free cosmic string spacetime one has the relation $\left\langle T_{0}^{0}\right\rangle_{\mathrm{s}}=\left\langle T_{3}^{3}\right\rangle_{\mathrm{s}}$. The latter is a consequence of the boost invariance along the axis of the cosmic string. For a massive field and for points near the string, $m r \ll 1$, the renormalized VEV diverges on the string as $r^{-4}$ with the leading term given by (4.16). At large distances, $m r \gg 1$, for $q<2$ the first term in the square brackets in (4.14) is absent and the dominant contribution in the second term comes from the region near the lower limit of the integration. In this case $\left\langle T_{\nu}^{\nu}\right\rangle_{\mathrm{s}}$ is suppressed by the factor $e^{-2 m r}$. For $q>2$ the dominant contribution at large distances comes from the $l=1$ term in (4.14) and the suppression factor is $e^{-2 m r \sin (\pi / q)}$. Note that the contribution of the first term in the square brackets of (4.14) is always negative for $\nu=0,1,3$, and positive for $\nu=2$. It can be easily checked that the boundary-free contribution (4.14) separately obeys the trace relation (4.5).

The contribution in (4.12), coming from the second term in the right-hand side of (4.4), is induced by the boundary at $z=0$ when the second boundary is absent. After the rotation of the integration contours in the integral (4.13) one gets

$$
\int_{0}^{\infty} d x e^{-2 y \sqrt{x^{2}+m^{2}}} \sum_{\eta= \pm 1} \frac{m e^{2 \eta i k z}}{m-\eta i k}=2 m G(2 m z, 2 m y)
$$

where

$$
G(2 m z, 2 m y)=\int_{m}^{\infty} d x \frac{e^{-2 x z}}{m+x} \sin \left(2 y \sqrt{x^{2}-m^{2}}\right) .
$$

An equivalent expression is obtained by using the representation $(m+x)^{-1}=\int_{0}^{\infty} d t e^{-(m+x) t}$. The integral over $x$ is expressed in terms of the Macdonald function and we find

$$
G(u, v)=v G_{1}(u, v) .
$$

Here and below we use the notation

$$
G_{\nu}(u, v)=e^{u} \int_{u}^{\infty} d x e^{-x} f_{\nu}\left(\sqrt{x^{2}+v^{2}}\right) .
$$

As a result, the VEV in the geometry of the single plate at $z=0$ is decomposed as (no summation over $\nu$ )

$$
\left\langle T_{\nu}^{\nu}\right\rangle^{(1)}=\left\langle T_{\nu}^{\nu}\right\rangle_{\mathrm{s}}+\left\langle T_{\nu}^{\nu}\right\rangle_{\mathrm{b}}^{(1)}
$$

where the boundary-induced contribution is given by

$$
\begin{aligned}
\left\langle T_{\nu}^{\nu}\right\rangle_{\mathrm{b}}^{(1)}= & \frac{2 m^{4}}{\pi^{2}}\left[\sum_{l=0}^{p}(-1)^{l} c_{l} F_{\nu}\left(2 m z, 2 m r s_{l}\right)+\frac{2 q}{\pi} \cos \left(\frac{q \pi}{2}\right)\right. \\
& \left.\times \int_{0}^{\infty} d x \frac{\sinh (q x) \sinh x}{\cosh (2 q x)-\cos (q \pi)} F_{\nu}(2 m z, 2 m r \cosh x)\right],
\end{aligned}
$$


with the functions

$$
\begin{aligned}
& F_{0}(x, y)=F_{1}(x, y)=-G_{2}(x, y) \\
& F_{2}(x, y)=2 G_{2}(x, y)+F(x, y)
\end{aligned}
$$

and $F_{3}(x, y)=0$. As we see, the boundary-induced part in the axial stress vanishes. Note that we have the relation

$$
F_{\nu}(2 m z, 2 m y)=\frac{1-\delta_{3}^{\nu}}{8 m^{3}} \hat{D}_{\nu} G(2 m z, 2 m y)
$$

By using this relation, we can see that in the limit $a \rightarrow \infty, z \rightarrow \infty$, with fixed $a-z$, from the second term in the right-hand side of (4.10) we obtain the boundary-induced part in the geometry of a single plate at $z=a$. The latter is given by (4.22) with the replacement $z \rightarrow a-z$.

By taking into account (4.24) and combining the last term in the right-hand side of (4.10) with the single plate-induced part (4.22), we get the following representation

$$
\left\langle T_{\nu}^{\nu}\right\rangle=\left\langle T_{\nu}^{\nu}\right\rangle_{\mathrm{s}}+\left\langle T_{\nu}^{\nu}\right\rangle_{\mathrm{b}}
$$

Here the boundary-free part is given by (4.14) and for the boundary-induced contribution in the region between the plates one has the expression

$$
\begin{aligned}
\left\langle T_{\nu}^{\nu}\right\rangle_{\mathrm{b}}= & \frac{1}{2 \pi^{2}}\left[\sum_{l=0}^{p}(-1)^{l} c_{l} C_{\nu}\left(z, r s_{l}\right)+\frac{2 q}{\pi} \cos \left(\frac{q \pi}{2}\right)\right. \\
& \left.\times \int_{0}^{\infty} d u \frac{\sinh (q u) \sinh (u) C_{\nu}(z, r \cosh u)}{\cosh (2 q u)-\cos (q \pi)}\right],
\end{aligned}
$$

with the notation

$$
C_{\nu}(z, y)=\hat{D}_{\nu} \int_{m}^{\infty} d x \frac{\sin \left(2 y \sqrt{x^{2}-m^{2}}\right)}{\frac{x+m}{x-m} e^{2 a x}+1}\left[1+\frac{m}{2}\left(1-\delta_{3}^{\nu}\right) \frac{e^{2 x z}+e^{2(a-z) x}}{x-m}\right] .
$$

As we could expect, in the region between the plates the VEVs are symmetric with respect to the plane $z=a / 2$. For a massless field one obtains

$$
C_{\nu}(z, y)=\frac{\pi}{4 a} \hat{D}_{\nu}\left[\frac{1}{\pi y / a}-\frac{1}{\sinh (\pi y / a)}\right]
$$

Note that in the latter case the single plate part in the VEV of the energy-momentum tensor vanishes and in the region between the plates the VEV of the energy-momentum tensor does not depend on the coordinate $z$.

It can be checked that the boundary-induced contribution obeys the covariant conservation equation $\nabla_{\mu}\left\langle T_{\nu}^{\mu}\right\rangle_{\mathrm{b}}=0$. For the geometry under consideration the latter is reduced to a single equation $\partial_{r}\left(r\left\langle T_{1}^{1}\right\rangle_{\mathrm{b}}\right)=\left\langle T_{2}^{2}\right\rangle_{\mathrm{b}}$. The latter equation directly follows from the relation

$$
\left(y \partial_{y}+1\right) \hat{D}_{1} \sin (b y)=\hat{D}_{2} \sin (b y) .
$$

By taking into account the relation (4.8) we can see that $\sum_{\nu=0}^{3} C_{\nu}(z, y)=m C(z, y)$. From here it follows that the boundary induced contributions obey the trace relation (4.5).

For the further transformation of the VEV (4.26) in the case of a massless field, we first separate the Minkowskian part (the $l=0$ term). It can be seen that the contribution of the first term in the square brackets of (4.28) to the remaining (topological) part of $\left\langle T_{\nu}^{\nu}\right\rangle_{\mathrm{b}}$ is equal 
to $-\left\langle T_{\nu}^{\nu}\right\rangle_{\mathrm{s}}$. Hence, in the total VEV, this contribution is cancelled by the boundary-free part and for the total VEV in the massless case we get

$$
\begin{aligned}
\left\langle T_{\nu}^{\nu}\right\rangle= & \left\langle T_{\nu}^{\nu}\right\rangle_{\mathrm{M}}-\frac{\pi^{2}}{8 a^{4}}\left[\sum_{l=1}^{p}(-1)^{l} c_{l} A_{\nu}\left(\pi s_{l} r / a\right)+\frac{2 q}{\pi} \cos \left(\frac{q \pi}{2}\right)\right. \\
& \left.\times \int_{0}^{\infty} d u \frac{\sinh (q u) \sinh (u) A_{\nu}(\pi(r / a) \cosh u)}{\cosh (2 q u)-\cos (q \pi)}\right]
\end{aligned}
$$

with the functions

$$
A_{\nu}(y)=\hat{D}_{\nu} \frac{1}{\sinh y} .
$$

For a massless field from (4.8) one has $\sum_{\nu=0}^{3} \hat{D}_{\nu}=0$ and both the Minkowskian and topological parts in (4.30) are traceless.

In the absence of the cosmic string, the only nonzero contribution to (4.26) comes from the term with $l=0$ and we obtain the VEVs in the region between two plates in Minkowski bulk (no summation over $\nu$ ):

$$
\begin{aligned}
\left\langle T_{\nu}^{\nu}\right\rangle_{\mathrm{M}} & =-\frac{2}{3 \pi^{2}} \int_{m}^{\infty} d x \frac{\left(x^{2}-m^{2}\right)^{3 / 2}}{\frac{x+m}{x-m} e^{2 a x}+1}\left[1+\frac{m}{2} \frac{e^{2 x z}+e^{2(a-z) x}}{x-m}\right], \\
\left\langle T_{3}^{3}\right\rangle_{\mathrm{M}} & =\frac{2}{\pi^{2}} \int_{m}^{\infty} d x \frac{x^{2} \sqrt{x^{2}-m^{2}}}{\frac{x+m}{x-m} e^{2 a x}+1}
\end{aligned}
$$

where $\nu=0,1,2$. In particular, the energy density is negative. For a massless field one has $\left\langle T_{3}^{3}\right\rangle_{\mathrm{M}}=-3\left\langle T_{0}^{0}\right\rangle_{\mathrm{M}}=7 \pi^{2} /\left(960 a^{4}\right)$. The fermionic Casimir densities for the geometry of two parallel plates in background of flat spacetime with an arbitrary number toroidally compactified spatial dimensions have been investigated in 23. The expressions (4.32) are special cases of the corresponding general formulas. The VEV of the energy-momentum tensor for parallel branes on AdS bulk is investigated in 24]. The fermionic Casimir energy for two parallel plates in 4-dimensional Minkowski spacetime has been studied in [29, 30]. The corresponding result for an arbitrary number of dimensions is generalized in [31. The influence of the compactification of spatial dimensions on the fermionic Casimir energy has been discussed in [32].

Let us consider the boundary-induced contribution in the VEV of the energy-momentum tensor in the asymptotic regions. For points outside the plates, the boundary-induced part is finite on the string. Taking the limit $r \rightarrow 0$ it can be seen that $C_{\nu}(z, 0)=4 \pi^{2}\left\langle T_{\nu}^{\nu}\right\rangle_{\mathrm{M}}$. Hence, from (4.26) one gets

$$
\left\langle T_{\nu}^{\nu}\right\rangle_{\mathrm{b}, r=0}=2\left\langle T_{\nu}^{\nu}\right\rangle_{\mathrm{M}}\left[\sum_{l=0}^{p}(-1)^{l} c_{l}+\frac{2 q}{\pi} \cos \left(\frac{q \pi}{2}\right) \int_{0}^{\infty} d x \frac{\sinh (q x) \sinh x}{\cosh (2 q x)-\cos (q \pi)}\right] .
$$

Note that the expression in the square brackets is the same as that in the corresponding formula (3.33) for the fermionic condensate. This expression is zero and, hence, the boundary-induced contribution in the VEV of the energy-momentum tensor vanishes on the string. This means that in the region near the cosmic string the VEV of the energy-momentum tensor is dominated by the boundary-free part and the corresponding energy density is negative.

In order to obtain the behavior of the vacuum energy-momentum tensor at large distances from the string, assuming that $m r \gg 1$, we need to find the asymptotic estimate for the function $C_{\nu}(z, y)$ for large values of $y$. In this limit the dominant contribution to the integral in (4.27) 
comes from the region near the lower limit of the integration and one gets

$$
\begin{aligned}
& C_{\nu}(z, y) \approx \frac{e^{2 m z}+e^{2 m(a-z)}}{4 m} \hat{D}_{\nu} \int_{m}^{\infty} d x x \sin \left(2 y \sqrt{x^{2}-m^{2}}\right) e^{-2 a x}, \\
& C_{3}(z, y) \approx \frac{1}{4} \hat{D}_{3} \int_{m}^{\infty} d x\left(x^{2} / m^{2}-1\right) \sin \left(2 y \sqrt{x^{2}-m^{2}}\right) e^{-2 a x},
\end{aligned}
$$

where $\nu=0,1,2$. The integrals are expressed in terms of the function $K_{\nu}\left(2 m \sqrt{a^{2}+y^{2}}\right)$. By using the corresponding asymptotic expression for large values of the argument, we find

$$
\begin{aligned}
& C_{\nu}(z, y) \approx-\sqrt{\pi} a m^{3 / 2}\left[e^{2 m z}+e^{2 m(a-z)}\right] \frac{e^{-2 m y}}{4 y^{7 / 2}} \\
& C_{3}(z, y) \approx-\sqrt{\pi} a m^{5 / 2} \frac{e^{-2 m y}}{2 y^{5 / 2}}
\end{aligned}
$$

for $\nu=0,1$ and $C_{2}(z, y) \approx-2 m y C_{0}(z, y)$. Substituting into (4.26), we see that for $q>2$ the dominant contribution comes from the $l=1$ term and for $m r \gg 1$ one gets:

$$
\begin{aligned}
\left\langle T_{\nu}^{\nu}\right\rangle_{\mathrm{b}} & \approx\left\langle T_{\nu}^{\nu}\right\rangle_{\mathrm{M}}+\frac{a m^{5}}{8 \pi^{3 / 2}}\left[e^{2 m z}+e^{2 m(a-z)}\right] \frac{\cos (\pi / q) e^{-2 m r \sin (\pi / q)}}{[m r \sin (\pi / q)]^{7 / 2}} \\
\left\langle T_{2}^{2}\right\rangle_{\mathrm{b}} & \approx\left\langle T_{2}^{2}\right\rangle_{\mathrm{M}}-\frac{a m^{5}}{4 \pi^{3 / 2}}\left[e^{2 m z}+e^{2 m(a-z)}\right] \frac{\cos (\pi / q) e^{-2 m r \sin (\pi / q)}}{[m r \sin (\pi / q)]^{5 / 2}} \\
\left\langle T_{3}^{3}\right\rangle_{\mathrm{b}} & \approx\left\langle T_{3}^{3}\right\rangle_{\mathrm{M}}+\frac{a m^{5}}{4 \pi^{3 / 2}} \frac{\cos (\pi / q) e^{-2 m r \sin (\pi / q)}}{[m r \sin (\pi / q)]^{5 / 2}}
\end{aligned}
$$

Note that for the boundary-free part (4.14) one has the large distance asymptotic

$$
\left\langle T_{\nu}^{\nu}\right\rangle_{\mathrm{s}} \approx-\frac{m^{4}}{4 \pi^{3 / 2}} \frac{\cos (\pi / q) e^{-2 m r \sin (\pi / q)}}{[m r \sin (\pi / q)]^{5 / 2}},
$$

for $\nu=0,1,3$ and $\left\langle T_{2}^{2}\right\rangle_{\mathrm{s}} \approx-3\left\langle T_{0}^{0}\right\rangle_{\mathrm{s}}$. In the case $1 \leqslant q \leqslant 2$ the suppression of the string-induced contribution is stronger, by the factor $e^{-2 m r}$.

For a massless field, the topological part in the VEV of the energy-momentum tensor is given by the second term in the right-hand side of (4.30). At large distances from the string, $r \gg a$, and for $q>2$ the dominant contribution to the topological part comes from the term with $l=1$. To the leading order we find

$$
\left\langle T_{\nu}^{\nu}\right\rangle \approx\left\langle T_{\nu}^{\nu}\right\rangle_{\mathrm{M}}+\frac{\pi A_{\nu}^{(0)}(\pi(r / a) \sin (\pi / q))}{4 a^{3} r e^{\pi(r / a) \sin (\pi / q)}} \cot (\pi / q)
$$

where

$$
A_{0}^{(0)}(y)=A_{1}^{(0)}(y)=\frac{1}{y}, A_{2}^{(0)}(y)=-A_{3}^{(0)}(y)=-1 .
$$

For $q \leqslant 2$, in the topological part of (4.30) the integral term remains only and the topological part falls off as $e^{-\pi r / a}$. For a massless field the boundary-free part $\left\langle T_{\nu}^{\nu}\right\rangle_{\mathrm{s}}$ decays as $1 / r^{4}$. Note that in the region between two plates the decay of the topological part at large distances from the string is exponential for both the massive and massless cases.

The VEVs $\left\langle T_{\nu}^{\nu}\right\rangle$ for $\nu=0,1,2$ diverge on the plates. This type of surface divergences are well known in quantum field theory with boundaries and have been investigated for various bulk and boundary geometries. In the problem under consideration, for points away from the cosmic string, $r \neq 0$, the divergences are the same as those for the plates in Minkowski bulk. 
This means that the part in the VEV induced by the cosmic string (the topological part), $\left\langle T_{\nu}^{\nu}\right\rangle_{\mathrm{t}}=\left\langle T_{\nu}^{\nu}\right\rangle-\left\langle T_{\nu}^{\nu}\right\rangle_{\mathrm{M}}$, is finite on the plates. This feature could be deduced from general arguments. Indeed, for points $r \neq 0$, both the local bulk and boundary geometries for the cosmic string and for Minkowski bulks are the same. The divergences are determined by the local geometrical characteristics (curvature tensors for the bulk and boundaries) and, consequently, they are the same as well. In order to find the leading terms in the asymptotic expansion of $\left\langle T_{\nu}^{\nu}\right\rangle, \nu=0,1,2$, for points near the plate at $z=0$, we note that the dominant contribution to the integral in (4.32) comes from large values of $x$. One can see that to the leading order $\left\langle T_{\nu}^{\nu}\right\rangle \approx-m /\left(12 \pi^{2} z^{3}\right)$. The axial stress is finite on the plates.

In figure 2 we have plotted the ratio of the boundary-induced parts in the energy density ( $\nu=0$, full curves) and the azimuthal stress $(\nu=2$, dashed curves $)$ to the corresponding quantities for parallel plates in Minkowski spacetime versus the distance from the cosmic string. The graphs are plotted for a massless fermionic field and the numbers near the curves are the values of the parameter $q$.

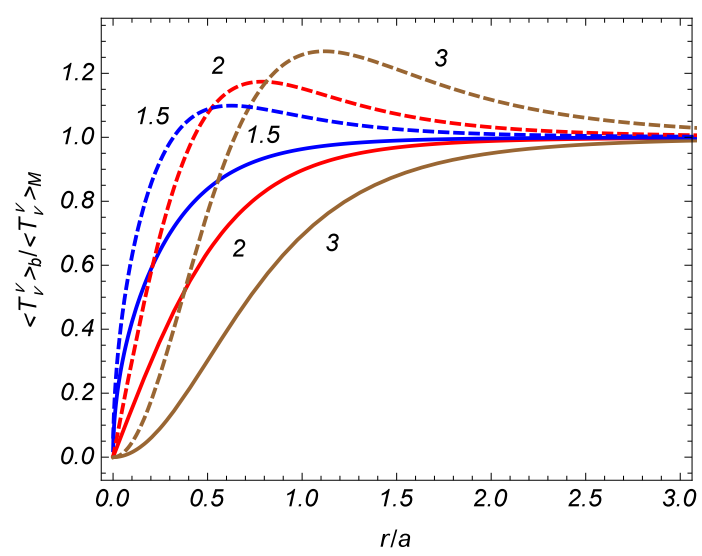

Figure 2: The ratio of the plate-induced contributions in the energy density (full curves) and in the azimuthal stress (dashed curves) to the corresponding quantities in the Minkowski bulk as functions of the distance from the string for a massless fermionic field. The numbers near the curves are the values of the parameter $q$.

We could apply the summation formula (3.12) to the series over $n$ in the formula (4.2). This gives the following equivalent representation for the diagonal components of the vacuum energy-momentum tensor (no summation over $\nu$ ):

$$
\begin{aligned}
\left\langle T_{\nu}^{\nu}\right\rangle= & \left\langle T_{\nu}^{\nu}\right\rangle_{\mathrm{s}}-\frac{q}{\pi^{2}} \sum_{j} \int_{0}^{\infty} d \lambda \lambda \bar{g}_{q j-1 / 2}^{(\nu)}(\lambda r) \\
& \times \int_{\sqrt{\lambda^{2}+m^{2}}}^{\infty} d x \frac{f^{(\nu)}(x, z)}{\frac{x+m}{x-m} e^{2 a x}+1}\left(x^{2}-\lambda^{2}-m^{2}\right)^{-1 / 2} .
\end{aligned}
$$

Here, we have defined the functions

$$
\bar{g}_{\beta}^{(0)}(y)=\bar{g}_{\beta}^{(3)}(y)=J_{\beta}^{2}(y)+J_{\beta+1}^{2}(y),
$$

$\bar{g}_{\beta}^{(\nu)}(y)=g_{\beta}^{(\nu)}(y)$ for $\nu=1,2$, and

$$
\begin{aligned}
& f^{(0)}(x, z)=\left(x^{2}-\lambda^{2}-m^{2}\right) f(x, z) \\
& f^{(1)}(x, z)=f^{(2)}(x, z)=\lambda^{2} f(x, z), f^{(3)}(x, z)=-2 x^{2}
\end{aligned}
$$


with

$$
f(x, z)=2+m \frac{e^{2 x z}+e^{2 x(a-z)}}{x-m} .
$$

The second term in the right-hand side of (4.40) presents the contribution induced by the boundaries. As it has been shown above, the radial stress is equal to the energy density. From (4.40) it follows that for $r \neq 0$ the boundary-induced contribution is negative to the energy density and positive for the axial stress. By taking into account that the boundary-free part in the energy density is negative as well, we conclude that the total energy density is negative everywhere, $\left\langle T_{0}^{0}\right\rangle<0$.

We have already shown that the boundary-induced VEVs vanish on the cosmic string. For points near the string, the contribution of the term $j=1 / 2$ into the boundary-induced part in (4.40) dominates and to the leading order we get (no summation over $\nu$ )

$$
\left\langle T_{\nu}^{\nu}\right\rangle \approx\left\langle T_{\nu}^{\nu}\right\rangle_{\mathrm{s}}-\frac{g_{\nu} \pi^{-3 / 2}(r / 2)^{q-1}}{(q+2) \Gamma(q / 2) \Gamma((q+1) / 2)} \int_{m}^{\infty} d x \frac{\left(x^{2}-m^{2}\right)^{q / 2+1}}{\frac{x+m}{x-m} e^{2 a x}+1} f(x, z),
$$

for $\nu=0,1,2$ with

$$
g_{0}=g_{1}=1, g_{2}=q
$$

and

$$
\left\langle T_{3}^{3}\right\rangle \approx\left\langle T_{3}^{3}\right\rangle_{\mathrm{s}}+\frac{2 \pi^{-3 / 2}(r / 2)^{q-1}}{\Gamma(q / 2) \Gamma((q+1) / 2)} \int_{m}^{\infty} d x \frac{x^{2}\left(x^{2}-m^{2}\right)^{q / 2}}{\frac{x+m}{x-m} e^{2 a x}+1} .
$$

In the special case $q=1$ these asymptotics are reduced to the exact results (4.32).

\section{Casimir force}

The Casimir force acting per unit surface of the plate is determined by the normal stress $\left\langle T_{3}^{3}\right\rangle$. For the corresponding effective pressure one has: $p=-\left\langle T_{3}^{3}\right\rangle$. The boundary free part of the pressure is the same on both the sides of the plate and it does not contribute to the net force. Hence, the force per unit surface of the plate is determined by the boundary-induced part of the pressure along the $z$-direction. By taking into account that $\left\langle T_{3}^{3}\right\rangle_{\mathrm{b}}^{(1)}=0$, one finds

$$
\begin{aligned}
p(r)= & p_{\mathrm{M}}-\frac{2}{\pi^{2}}\left[\sum_{l=1}^{p}(-1)^{l} c_{l} F\left(r s_{l}\right)+\frac{2 q}{\pi} \cos \left(\frac{q \pi}{2}\right)\right. \\
& \left.\times \int_{0}^{\infty} d x \frac{\sinh (q x) \sinh (x) F(r \cosh x)}{\cosh (2 q x)-\cos (q \pi)}\right],
\end{aligned}
$$

with the function

$$
F(y)=\frac{1}{y} \int_{m}^{\infty} d x \frac{x^{2} \sin \left(2 y \sqrt{x^{2}-m^{2}}\right)}{\frac{x+m}{x-m} e^{2 a x}+1} .
$$

Here $p_{\mathrm{M}}=-\left\langle T_{3}^{3}\right\rangle_{\mathrm{M}}$, with $\left\langle T_{3}^{3}\right\rangle_{\mathrm{M}}$ from (4.32), is the fermionic Casimir pressure for the plates in Minkowski bulk. From the results of the previous section it follows that for $q>1$ the Casimir pressure vanishes on the string as $r^{q-1}$. An alternative representation for the Casimir pressure is obtained by using the formula (4.40) for the axial stress:

$$
\begin{aligned}
p(r)= & -\frac{2 q}{\pi^{2}} \sum_{j} \int_{0}^{\infty} d \lambda \lambda\left[J_{q j-1 / 2}^{2}(\lambda r)+J_{q j+1 / 2}^{2}(\lambda r)\right] \\
& \times \int_{\sqrt{\lambda^{2}+m^{2}}}^{\infty} d x x^{2} \frac{\left(x^{2}-\lambda^{2}-m^{2}\right)^{-1 / 2}}{\frac{x+m}{x-m} e^{2 a x}+1} .
\end{aligned}
$$


For $r \neq 0$ this pressure is always negative which means that the Casimir forces are attractive.

At large distances from the string and for a massive field, $m r \gg 1$, from (4.36) for $q>2$ it follows that

$$
p(r) \approx p_{\mathrm{M}}-\frac{a m^{5}}{4 \pi^{3 / 2}} \frac{\cos (\pi / q) e^{-2 m r \sin (\pi / q)}}{[m r \sin (\pi / q)]^{5 / 2}} .
$$

For $q<2$ and if $q$ is not too close to 2 one has

$$
p \approx p_{M}+\frac{q^{2} m^{3} \cos (q \pi / 2) e^{-2 m r}}{8 \pi^{2} \sin ^{2}(q \pi / 2)(m r)^{3}},
$$

with the exponential suppression of the topological part.

For a massless field, in (5.1) one has $p_{\mathrm{M}}=-7 \pi^{2} /\left(960 a^{4}\right)$ and

$$
\begin{aligned}
F(y) & =-\frac{\pi}{16 a y} \partial_{y}^{2}\left[\frac{a}{\pi y}-\frac{1}{\sinh (y \pi / a)}\right] \\
& =-\frac{1}{8 y^{4}}\left[1-\left(\frac{y \pi}{a}\right)^{3} \frac{1+\sinh ^{2}(y \pi / a) / 2}{\sinh ^{3}(y \pi / a)}\right] .
\end{aligned}
$$

At large distances from the string, this leads to the following asymptotic:

$$
p \approx-\frac{7 \pi^{2}}{960 a^{4}}\left[1+\frac{\left(q^{2}-1\right)\left(q^{2}+17 / 7\right)}{3 \pi^{4}(r / a)^{4}}\right], r \gg a .
$$

In this case one has a power law decay for the topological part. As it has been shown before, for a massless field and at large distances from the string the topological part of the axial stress in the region between the plates is suppressed by the factor $e^{-\pi(r / a) \sin (\pi / q)}$ for $q>2$ and by $e^{-\pi r / a}$ for $q \leqslant 2$. In (5.7), the leading term in the topological part (the second term in the square brackets) comes from the pressure in the exterior region $(z \leqslant 0$ for the plate at $z=0$ and $z \geqslant a$ for the plate at $z=a$ ). The latter is dominated by the boundary-free part and induces an attractive force.

Let us denote by $\varepsilon_{\mathrm{t}}$ the topological part in the vacuum energy per unit surface of the plates:

$$
\varepsilon_{\mathrm{t}}=\frac{1}{\phi_{0}} \int_{0}^{a} d z \int_{0}^{\phi_{0}} d \phi\left\langle T_{0}^{0}\right\rangle_{\mathrm{t}} .
$$

where the topological contribution to the energy density is obtained from (4.25) omitting the $l=0$ term (corresponding to $\left\langle T_{0}^{0}\right\rangle_{\mathrm{M}}$ ) in the boundary-induced part (4.26). Note that $\varepsilon_{\mathrm{t}}$ depends on the radial coordinate $r$. Integrating by parts, one can see that

$$
\partial_{a} \int_{0}^{a} d z C_{0}(z, y)=4 F(y) .
$$

From here the following relation is obtained between the topological parts in the vacuum energy and the pressure on the plates:

$$
p_{\mathrm{t}}=-\partial_{a} \varepsilon_{\mathrm{t}}
$$

where $p_{\mathrm{t}}$ is given by the second term in the right-hand side of (5.1). This is the standard thermodynamical relation between the energy and pressure for adiabatic processes. Note that the Minkowkian part $\left\langle T_{0}^{0}\right\rangle_{\mathrm{M}}$ diverges on the plates and in order to obtain the finite vacuum energy an additional renormalization is required.

Figure 3 presents the ratio of the Casimir force per unit surface of the plate to the corresponding quantity in the Minkowski spacetime as a function of the distance from the cosmic string. The left panel is plotted for a massless field and for the right panel we have taken $m a=0.5$. In the latter case for the Minkowskian pressure one has $p_{\mathrm{M}} \approx-0.0384 / a^{4}$. The numbers near the curves are the values of the parameter $q$. As is seen from the right panel, the dependence, in general, is not monotonic. 

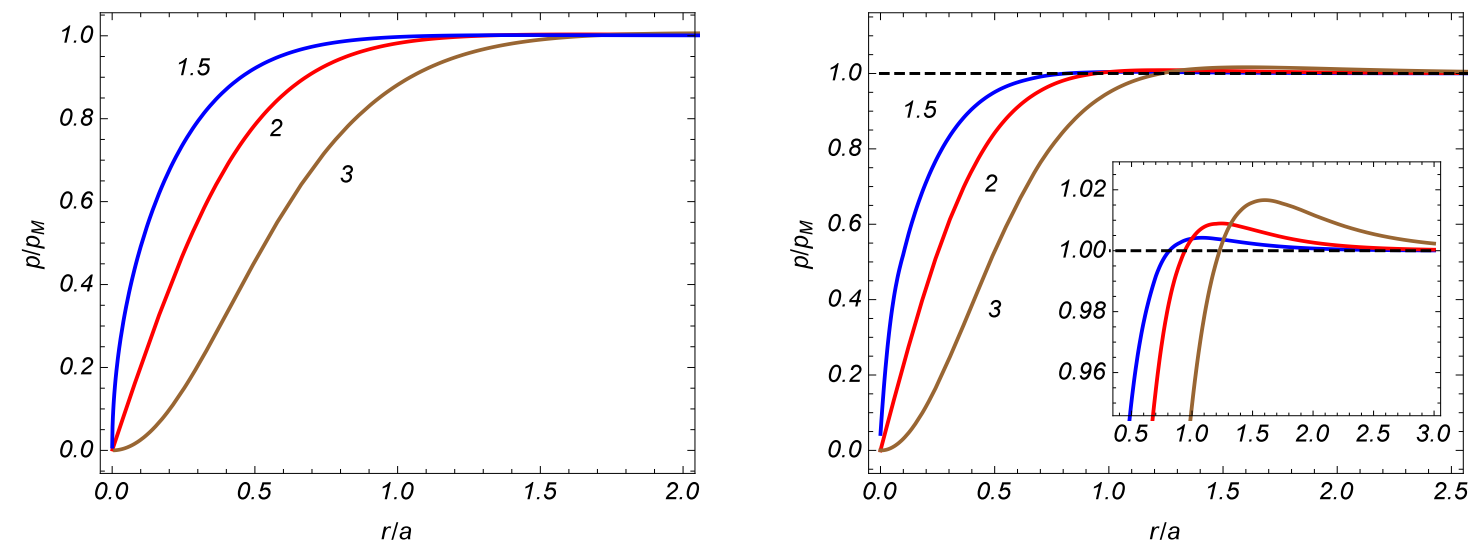

Figure 3: The ratio of the Casimir pressure to the corresponding quantity in the Minkowski bulk versus the distance from the string. The left panel is for a massless field and for the right panel $m a=0.5$. The numbers near the curves are the values of the parameter $q$.

\section{Conclusion}

We have investigated the influence of the conical geometry on the characteristics of the fermionic vacuum in the region between two plates perpendicular to the cone axis. This geometry describes an idealized cosmic string with zero thickness core. On the plates the field obeys the boundary condition used in bag models to confine the quarks inside the hadrons. Among the most important local characteristics of the fermionic vacuum are the fermionic condensate and the expectation value of the energy-momentum tensor. For the evaluation of the corresponding VEVs we have constructed the complete set of positive- and negative-energy fermionic modes, given by the expressions (2.5) and (2.11). The eigenvalues of the quantum number, corresponding to the direction along the cosmic string axis, are quantized by the boundary conditions and are solutions of the transcendental equation (2.10). The mode sums of the VEVs contain series over these eigenvalues. The application of the Abel-Plana type formula (3.12) allows to extract the parts in the VEVs for the geometry of a single plate and to present the second plate induced contributions in the form for which the explicit knowledge of the eigenvalues of the axial quantum number is not required.

The fermionic condensate is decomposed into the boundary-free and boundary-induced contributions (see (3.25)). The boundary-free part is given by (3.20) and it vanishes for a massless field. For the boundary-induced part we have derived the expression (3.26) with the function $C(z, y)$ defined as (3.27). The corresponding integral is further evaluated for a massless field with the expression (3.28). The $l=0$ term in the boundary-induced contribution (3.26) corresponds to the fermion condensate for two parallel plates in Minkowski bulk. The remaining part is induced by the nontrivial topology of the cosmic string. We have shown that, for points outside the string core, the topological part vanishes on the plates as a consequence of the cancellation between the boundary-free and boundary-induced parts. The Minkowskian part diverges on the plates with the leading term inversely proportional to the cube of the distance from the plate. For points away from the plates, the boundary-induced fermionic condensate vanishes on the string as $r^{q-1}$. For $q>2$, at large distances from the cosmic string the topological part in the boundary-induced fermionic condensate decays as $e^{-2 m r \sin (\pi / q)}$ for a massive field and as $e^{-2 r \sin (\pi / q) / a}$ for a massless field. In the case $q \leqslant 2$ the decay is stronger, like $e^{-2 m r}$ and $e^{-2 r / a}$ for the massive and massless cases, respectively. An alternative representation for the 
boundary-induced contribution to the fermionic condensate is given by the second term in the right-hand side of (3.38). This contribution is negative for points outside the cosmic string. For points near the cosmic string the fermionic condensate is dominated by the boundary-free part and is positive. At large distances from the string, the Minkowskian part dominates and the condensate is negative. Consequently, at some intermediate distances the fermionic condensate vanishes.

The vacuum energy-momentum tensor is diagonal and the corresponding radial stress is equal to the energy density. The boundary-induced contributions to the separate components are given by (4.26), where the functions $C_{\nu}(z, y)$ are defined by the expressions (4.27) and (4.28) for massive and massless fields respectively. In the latter case the single plate parts vanish and the VEV of the energy-momentum tensor in the region between the plates does not depend on the coordinate $z$. For the axial stress, that is the case for a massive field as well. Similar to the fermionic condensate, for points away the plates, the boundary-induced contributions to the vacuum energy-momentum tensor vanish on the string. At distances from the cosmic string larger than the Compton wavelength, $m r \gg 1$, the asymptotic for the topological parts is given by (4.36) and they are suppressed by the factor $e^{-2 m r \sin (\pi / q)}$ for $q>2$ and by the factor $e^{-2 m r}$ for $q \leqslant 2$. Compared with the azimuthal and axial stresses, the topological contributions to the energy density and the radial stress contain an additional suppression factor $1 /(\mathrm{mr})$. For a massless field and at distances $r \gg a$, one has the asymptotic behavior (4.38) with the suppression factor $e^{-2 r \sin (\pi / q) / a}$ for $q>2$ and $e^{-2 r / a}$ for $q \leqslant 2$. This behavior for the topological part is in contrast to that for the boundary-free geometry. In the latter case the decay of the vacuum energy-momentum tensor is power law, as $1 / r^{4}$. Another representation for the VEV of the energy-momentum tensor in the region between the boundaries is given by (4.40) with the last term been the boundary-induced contribution. Both the boundary-free and boundaryinduced contributions to the energy density are negative.

We have also considered the Casimir force per unit surface of the plates (the vacuum effective pressure), given by (5.1). It is decomposed into the Minkowskian and topological parts, the latter being induced by the cosmic string. The vacuum pressure on the plates is not homogeneous. It vanishes at the point where the cosmic string crosses the plates and is negative at other points. This corresponds to the attractive force between the plates. The Casimir pressure on the plates, in general, is not a monotonic function of the distance from the string. We have shown that the topological contributions in the vacuum energy and the pressure obey the standard thermodynamical relation (5.10).

\section{Acknowledgments}

A.A.S. was supported by the State Committee of Science Ministry of Education and Science RA, within the frame of Grant No. SCS 15T-1C110, and by the Armenian National Science and Education Fund (ANSEF) Grant No. hepth-4172. 


\section{A Transformations for the energy-momentum tensor}

Here we describe some details for the presentation of the VEVs (4.2) in the form (4.6). Let us start with the energy density. The corresponding mode sum (4.1) is rewritten in the form

$$
\begin{aligned}
\left\langle T_{0}^{0}\right\rangle= & -\frac{q}{\pi a} \sum_{n=1}^{\infty} \frac{h_{0}\left(x_{n} / a, z\right)}{1-\sin \left(2 x_{n}\right) /\left(2 x_{n}\right)} \\
& \times \sum_{j} \int_{0}^{\infty} d \lambda \lambda \omega\left[J_{q j-1 / 2}^{2}(\lambda r)+J_{q j+1 / 2}^{2}(\lambda r)\right],
\end{aligned}
$$

with the notation $h_{0}(k, z)$ defined in accordance with (4.4). For the further transformation we use the integral representation $\omega=-(2 / \sqrt{\pi}) \int_{0}^{\infty} d s \partial_{s^{2}} e^{-\omega^{2} s^{2}}$. Integrating over $\lambda$ with the help of (3.6) and after the integration by parts in the integral over $s$, we get

$$
\begin{aligned}
\left\langle T_{0}^{0}\right\rangle= & \frac{\pi^{-3 / 2} q}{\sqrt{2} r^{3} a} \sum_{n=1}^{\infty} \frac{h_{0}\left(x_{n} / a, z\right)}{1-\sin \left(2 x_{n}\right) /\left(2 x_{n}\right)} \\
& \times \int_{0}^{\infty} d y y^{1 / 2} e^{-\left(x_{n}^{2} / a^{2}+m^{2}\right) r^{2} / 2 y-y} \mathcal{I}(q, y) .
\end{aligned}
$$

As the next step we employ the integral representation (3.9). After the integration over $y$, for the energy density this gives

$$
\begin{aligned}
\left\langle T_{0}^{0}\right\rangle= & -\frac{1}{4 \pi a r} \partial_{r} \frac{1}{r} \sum_{n=1}^{\infty} \frac{h_{0}\left(x_{n} / a, z\right)}{1-\sin \left(2 x_{n}\right) /\left(2 x_{n}\right)}\left[\sum_{l=0}^{p}(-1)^{l} \frac{c_{l}}{s_{l}^{3}} e^{-2 r s_{l} \sqrt{x_{n}^{2} / a^{2}+m^{2}}}\right. \\
& \left.+\frac{2 q}{\pi} \cos \left(\frac{q \pi}{2}\right) \int_{0}^{\infty} d u \frac{\sinh (q u) \sinh u}{\cosh (2 q u)-\cos (q \pi)} \frac{e^{-2 r \sqrt{x_{n}^{2} / a^{2}+m^{2}} \cosh u}}{\cosh ^{3} u}\right] .
\end{aligned}
$$

This representation is in the form (4.6).

Now let us consider the VEV of the azimuthal stress. By taking into account the relation

$$
J_{\nu}(\lambda r) J_{\nu+1}(\lambda r)=\left(\frac{\nu}{r}-\frac{1}{2} \frac{d}{d r}\right) \frac{1}{\lambda} J_{\nu}^{2}(\lambda r),
$$

for the Bessel function, the corresponding expression is presented in the form

$$
\begin{aligned}
\left\langle T_{2}^{2}\right\rangle= & -\frac{2 q^{2}}{\pi a r} \sum_{n=1}^{\infty} \frac{h_{2}\left(x_{n} / a, z\right)}{1-\sin \left(2 x_{n}\right) /\left(2 x_{n}\right)} \\
& \times \sum_{j} j\left(\frac{q j-1 / 2}{r}-\frac{1}{2} \partial_{r}\right) \int_{0}^{\infty} d \lambda \frac{\lambda}{\omega} J_{q j-1 / 2}^{2}(\lambda r) .
\end{aligned}
$$

By using the integral representation (3.5), after the integration over $\lambda$, the VEV is rewritten as

$$
\begin{aligned}
\left\langle T_{2}^{2}\right\rangle= & \frac{4 q^{2} r^{-3}}{(2 \pi)^{3 / 2} a} \sum_{n=1}^{\infty} \frac{h_{2}\left(x_{n} / a, z\right)}{1-\sin \left(2 x_{n}\right) /\left(2 x_{n}\right)} \sum_{j} j \int_{0}^{\infty} d y \\
& \times y^{-1 / 2} e^{-\left(k^{2}+m^{2}\right) r^{2} / 2 y}\left(q j-1 / 2-y \partial_{y}\right) e^{-y} I_{q j-1 / 2}(y) .
\end{aligned}
$$

Now, with the help of the relation

$$
\left(q j-1 / 2-y \partial_{y}\right) e^{-y} I_{q j-1 / 2}(y)=\frac{y}{q j} e^{-y}\left(y \partial_{y}-y+1 / 2\right)\left[I_{q j-1 / 2}(y)+I_{q j+1 / 2}(y)\right],
$$


the series over $j$ is expressed in terms of the function $\mathcal{I}(q, y)$ :

$$
\begin{aligned}
\left\langle T_{2}^{2}\right\rangle= & \frac{2 q r^{-3}}{(2 \pi)^{3 / 2} a} \sum_{n=1}^{\infty} \frac{h_{2}\left(x_{n} / a, z\right)}{1-\sin \left(2 x_{n}\right) /\left(2 x_{n}\right)} \int_{0}^{\infty} d y \\
& \times e^{-\left(k^{2}+m^{2}\right) r^{2} / 2 y-y} y^{1 / 2}\left(y \partial_{y}-y+1 / 2\right) \mathcal{I}(q, y) .
\end{aligned}
$$

By making use of the representation (3.9) we get

$$
\begin{aligned}
\left\langle T_{2}^{2}\right\rangle= & \frac{-1}{2 \pi a r^{3}}\left(1-r \partial_{r}+\frac{1}{2} r^{2} \partial_{r}^{2}\right) \sum_{n=1}^{\infty} \frac{h_{2}\left(x_{n} / a, z\right)}{1-\sin \left(2 x_{n}\right) /\left(2 x_{n}\right)}\left[\sum_{l=0}^{p}(-1)^{l} \frac{c_{l}}{s_{l}^{3}} e^{-2 r s_{l} \sqrt{x_{n}^{2} / a^{2}+m^{2}}}\right. \\
& \left.+\frac{2 q}{\pi} \cos \left(\frac{q \pi}{2}\right) \int_{0}^{\infty} d x \frac{\sinh (q x) \sinh x}{\cosh (2 q x)-\cos (q \pi)} \frac{e^{-2 r \sqrt{x_{n}^{2} / a^{2}+m^{2}} \cosh x}}{\cosh ^{3} x}\right]
\end{aligned}
$$

For the axial stress the mode sum is reduced to

$$
\left\langle T_{3}^{3}\right\rangle=\frac{q}{\pi a^{3}} \sum_{j} \int_{0}^{\infty} d \lambda \lambda \sum_{n=1}^{\infty} \frac{x_{n}^{2}}{\omega_{n}} \frac{J_{q j-1 / 2}^{2}(\lambda r)+J_{q j+1 / 2}^{2}(\lambda r)}{1-\sin \left(2 x_{n}\right) /\left(2 x_{n}\right)} .
$$

With the help of (3.5), after the integration over $\lambda$, we find

$$
\left\langle T_{3}^{3}\right\rangle=\frac{q a^{-3}}{\sqrt{2} \pi^{3 / 2} r} \sum_{n=1}^{\infty} \frac{x_{n}^{2}}{1-\sin \left(2 x_{n}\right) /\left(2 x_{n}\right)} \int_{0}^{\infty} d y y^{-1 / 2} e^{-\left(m^{2}+k^{2}\right) r^{2} / 2 y-y} \mathcal{I}(q, y) .
$$

By taking into account (3.9) and integrating over $y$ one gets the representation

$$
\begin{aligned}
\left\langle T_{3}^{3}\right\rangle= & \frac{1}{\pi a^{3} r} \sum_{n=1}^{\infty} \frac{x_{n}^{2}}{1-\sin \left(2 x_{n}\right) /\left(2 x_{n}\right)}\left[\sum_{l=0}^{p} \frac{(-1)^{l} \cot (\pi l / q)}{e^{2 r s_{l} \sqrt{x_{n}^{2} / a^{2}+m^{2}}}}\right. \\
& \left.+\frac{2 q}{\pi} \cos \left(\frac{q \pi}{2}\right) \int_{0}^{\infty} d x \frac{\sinh (q x) \tanh x}{\cosh (2 q x)-\cos (q \pi)} e^{-2 r \sqrt{x_{n}^{2} / a^{2}+m^{2}} \cosh x}\right] .
\end{aligned}
$$

The expression for the radial stress is obtained from the trace relation (4.5).

\section{References}

[1] V.M. Mostepanenko and N.N. Trunov, The Casimir Effect and its Applications (Clarendon, Oxford, 1997); E. Elizalde, S.D. Odintsov, A. Romeo, A.A. Bytsenko and S. Zerbini, Zeta Regularization Techniques with Applications (World Scientific, Singapore, 1994); K.A. Milton, The Casimir Effect: Physical Manifestation of Zero-Point Energy (World Scientific, Singapore, 2002); M. Bordag, G.L. Klimchitskaya, U. Mohideen, and V.M. Mostepanenko, Advances in the Casimir Effect (Oxford University Press, Oxford, 2009); Lecture Notes in Physics: Casimir Physics, Vol. 834, edited by D. Dalvit, P. Milonni, D. Roberts, and F. da Rosa (Springer, Berlin, 2011).

[2] E.R. Bezerra de Mello, A.A. Saharian, and M.R. Setare, Phys. Rev. D 92, 104005 (2015).

[3] A. Vilenkin and E.P.S. Shellard, Cosmic Strings and Other Topological Defects (Cambridge University Press, Cambridge, England, 1994); M.B. Hindmarsh and T.W.B. Kibble, Rep. Prog. Phys. 58, 477 (1995). 
[4] S. Sarangi and S.H.H. Tye, Phys. Lett. B 536, 185 (2002); E.J. Copeland, R.C. Myers, and J. Polchinski, J. High Energy Phys. 06 (2004) 013; G. Dvali and A. Vilenkin, J. Cosmol. Astropart. Phys. 03 (2004) 010.

[5] D.R. Nelson, Defects and Geometry in Condensed Matter Physics (Cambridge University Press, Cambridge, 2002); G.E. Volovik, The Universe in a Helium Droplet (Clarendon Press, Oxford, 2003).

[6] A. Krishnan, et al, Nature 388, 451 (1997); S.N. Naess, A. Elgsaeter, G. Helgesen, and K.D. Knudsen, Sci. Technol. Adv. Mater. 10, 065002 (2009).

[7] S. Bellucci, E.R. Bezerra de Mello, A. de Padua, and A.A. Saharian, Eur. Phys. J. C 74, 2688 (2014).

[8] E.R. Bezerra de Mello, V.B. Bezerra, A.A. Saharian, and A.S. Tarloyan, Phys. Rev. D 74, 025017 (2006).

[9] V.V. Nesterenko and I.G. Pirozhenko, Class. Quantum Grav. 28, 175020 (2011).

[10] E.R. Bezerra de Mello, V.B. Bezerra, A.A. Saharian, and A.S. Tarloyan, Phys. Rev. D 78, 105007 (2008).

[11] E.R. Bezerra de Mello, V.B. Bezerra, A.A. Saharian, and V.M. Bardeghyan, Phys. Rev. D 82, 085033 (2010); S. Bellucci, E.R. Bezerra de Mello, and A.A. Saharian, Phys. Rev. D. 83, 085017 (2011); E.R. Bezerra de Mello, F. Moraes, and A.A. Saharian, Phys. Rev. D 85, 045016 (2012).

[12] I. Brevik and T. Toverud, Class. Quantum Grav. 12, 1229 (1995).

[13] E.R. Bezerra de Mello, V.B. Bezerra, and A.A. Saharian, Phys. Lett. B 645, 245 (2007).

[14] G. Fucci and K. Kirsten, J. High Energy Phys. 03(2011)016; G. Fucci and K. Kirsten, J. Phys. A: Math. Theor. 44, 295403 (2011).

[15] E.R. Bezerra de Mello and A.A. Saharian, Class. Quantum Grav. 28, 145008 (2011).

[16] E.R. Bezerra de Mello, A.A. Saharian, and A.Kh. Grigoryan, J. Phys. A: Math. Theor. 45, 374011 (2012).

[17] E.R.Bezerra de Mello, A.A. Saharian, and S.V. Abajyan, Class. Quantum Grav. 30, 015002 (2013).

[18] K. Higashijima, Prog. Theor. Phys. Suppl. 104, 1 (1991); B. Rosenstein, B. J. Warr and S. H. Park, Phys. Rep. 205, 59 (1991).

[19] T. Inagaki, T. Muta, and S.D. Odintsov, Prog. Theor. Phys. Suppl. 127, 93 (1997).

[20] E.R. Bezerra de Mello and A.A. Saharian, Class. Quantum Grav. 29, 035006 (2012); E.R. Bezerra de Mello and A.A. Saharian, Eur. Phys. J. C 73, 2532 (2013).

[21] A.P. Prudnikov, Yu.A. Brychkov, and O.I. Marichev, Integrals and Series (Gordon and Breach, New York, 1986), Vol. 2.

[22] A.A. Saharian, The Generalized Abel-Plana Formula with Applications to Bessel Functions and Casimir Effect (Yerevan State University Publishing House, Yerevan, 2008) (arXiv:0708.1187). 
[23] E. Elizalde, S.D. Odintsov, and A.A. Saharian, Phys. Rev. D 83, 105023 (2011).

[24] E. Elizalde, S.D. Odintsov, and A.A. Saharian, Phys. Rev. D 87, 084003 (2013).

[25] M.O. Ribas, F.P. Devecchi, and G.M. Kremer, Europhys. Lett. 81, 19001 (2008); R.C. de Souza and G. M. Kremer, Class. Quant. Grav. 25, 225006 (2008).

[26] D. Ebert and K. G. Klimenko, Phys. Rev. D 82, 025018 (2010); A. Flachi, Phys. Rev. D 86, 104047 (2012); A. Flachi, Phys. Rev. Lett. 110, 060401 (2013); A. Flachi, Phys. Rev. D 88, 085011 (2013).

[27] V.B. Bezerra and N.R. Khusnutdinov, Class. Quantum Grav. 23, 3449 (2006).

[28] V.P. Frolov and E.M. Serebriany, Phys. Rev. D 35, 3779 (1987); J.S. Dowker, Phys. Rev. D 36, 3742 (1987).

[29] K. Johnson, Acta Phys. Pol. B 6, 865 (1975).

[30] S.G. Mamaev and N.N. Trunov, Sov. Phys. J. 23, 551 (1980).

[31] R.D.M. De Paola, R.B. Rodrigues, and N.F. Svaiter, Mod. Phys. Lett. A 14, 2353 (1999); E. Elizalde, F.C. Santos, and A.C. Tort, Int. J. Mod. Phys. A 18, 1761 (2003).

[32] S. Bellucci and A. A. Saharian, Phys. Rev. D 80, 105003 (2009). 